\title{
Drosophila Neuroligin 2 is Required Presynaptically and Postsynaptically for Proper Synaptic Differentiation and Synaptic Transmission
}

\author{
Yu-Chi Chen, ${ }^{1,2}$ Yong Qi Lin, ${ }^{5}$ Swati Banerjee, ${ }^{2}$ Koen Venken, ${ }^{5}$ Jingjun Li, ${ }^{2,4}$ Afshan Ismat, ${ }^{2}$ Kuchuan Chen, ${ }^{5}$ \\ Lita Duraine, ${ }^{5}$ Hugo J. Bellen, ${ }^{5}$ and Manzoor A. Bhat ${ }^{1,2,3,4}$ \\ ${ }^{1}$ Curriculum in Genetics and Molecular Biology, ${ }^{2}$ Department of Cell and Molecular Physiology, ${ }^{3}$ Curriculum in Neurobiology, and ${ }^{4}$ UNC-Neuroscience \\ Center, and Carolina Institute for Developmental Disabilities, University of North Carolina School of Medicine, Chapel Hill, North Carolina 27599-7545; \\ ${ }^{5}$ Howard Hughes Medical Institute, Department of Molecular and Human Genetics, Department of Neuroscience, Program in Developmental Biology, \\ Neurological Research Institute at Baylor College of Medicine, Houston, Texas 77030
}

Trans-synaptic adhesion between Neurexins (Nrxs) and Neuroligins (Nlgs) is thought to be required for proper synapse organization and modulation, and mutations in several human Nlgs have shown association with autism spectrum disorders. Here we report the generation and phenotypic characterization of Drosophila neuroligin $2(d n l g 2)$ mutants. Loss of dnlg2 results in reduced bouton numbers, aberrant presynaptic and postsynaptic development at neuromuscular junctions (NMJs), and impaired synaptic transmission. In dnlg2 mutants, the evoked responses are decreased in amplitude, whereas the total active zone (AZ) numbers at the NMJ are comparable to wild type, suggesting a decrease in the release probability. Ultrastructurally, the presynaptic AZ number per bouton area and the postsynaptic density area are both increased in $d n l g 2$ mutants, whereas the subsynaptic reticulum is reduced in volume. We show that both presynaptic and postsynaptic expression of Dnlg2 is required to restore synaptic growth and function in $d n l g 2$ mutants. Postsynaptic expression of Dnlg2 in dnlg2 mutants and wild type leads to reduced bouton growth whereas presynaptic and postsynaptic overexpression in wild-type animals results in synaptic overgrowth. Since Nlgs have been shown to bind to Nrxs, we created double mutants. These mutants are viable and display phenotypes that closely resemble those of $d n \lg 2$ and $d n r x$ single mutants. Our results provide compelling evidence that Dnlg2 functions both presynaptically and postsynaptically together with Neurexin to determine the proper number of boutons as well as the number of AZs and size of synaptic densities during the development of NMJs.

\section{Introduction}

Synapses are the fundamental units of neural networks and exhibit tightly apposed presynaptic and postsynaptic areas that are enriched in cell adhesion molecules (Giagtzoglou et al., 2009). A

Received April 5, 2012; revised Sept. 6, 2012; accepted Sept. 13, 2012.

Author contributions:Y.-C.C., Y.Q.L., S.B., H.J.B., and M.A.B. designed research; Y.-C.C., Y.Q.L., S.B., K.V., J.L., A.I., K.C., and L.D. performed research; Y.-C.C., Y.Q.L., S.B., K.V., J.L., H.J.B., and M.A.B. analyzed data; Y.-C.C., Y.Q.L., S.B., H.J.B., and M.A.B. wrote the paper.

K.V. was supported by training grant T32 GM007526 to the Molecular and Human Genetics Department at Baylor College of Medicine. H.J.B. is an investigator of the Howard Hughes Medical Institute. This work was supported by the Simons Foundation Grant (SF-177037) and in part by the National Institutes of Health Grant NS050356 (M.A.B.) We are grateful to Aaron DiAntonio for anti-GluRIII, Gabrielle L. Boulianne, and Wei Xie for $d n l g 2^{K 070}$ and UAS-dnlg2, and Vivian Budnik for isogenized $w^{1118}$ fly stocks. We thank the Bloomington Stock Center for fly stocks, the Developmental Studies Hybridoma Bank, and the University of lowa for monoclonal antibodies; Michael Chua for assistance with GluR quantification; Rosa Mino for assistance with dnlg2 in situ hybridization; and Alan Fanning and past and current members of the Bhat laboratory for technical assistance and helpful discussions.

The authors declare no competing financial interests.

Correspondence should be addressed to Manzoor A. Bhat, Department of Physiology, University of Texas Health Science Center School of Medicine, 7703 Floyd Curl Drive, San Antonio, Texas 78229-3900. E-mail: bhatm@uthscsa.edu.

M. A. Bhat's and S. Banerjee's present address: Department of Physiology, University of Texas Health Science Center, School of Medicine, 7703 Floyd Curl Drive, San Antonio, Texas 78229-3900

A. Ismat's present address: Department of Cell Biology, Johns Hopkins University School of Medicine, Baltimore, MD 21205

DOI:10.1523/JNEUROSCI.1685-12.2012

Copyright $\odot 2012$ the authors $\quad 0270-6474 / 12 / 3216018-13 \$ 15.00 / 0$ group of synaptic adhesion proteins thought to orchestrate formation of the presynaptic and postsynaptic structures are the Neuroligins (Nlgs) and their binding partners Neurexins (Nrxs) (Craig and Kang, 2007; Südhof, 2008). A growing body of evidence associates these molecules with autism spectrum disorders (ASD), as mutations in human NLGs were discovered in ASD patients (Jamain et al., 2003; Szatmari et al., 2007). Nlgs are a family of transmembrane proteins with an extracellular domain that displays homology to acetylcholinesterase (AChE) and localizes to the postsynaptic membranes (Ichtchenko et al., 1995; Song et al., 1999). Nlgs form dimers and bind to Nrxs through this AChE-like domain. At the C terminus, Nlgs have a PSD-95, Dlg, and ZO-1 (PDZ) domain binding sequence motif, which can interact with PDZ domain containing proteins (Song et al., 1999; Nourry et al., 2003) such as PSD-95 (Irie et al., 1997; Iida et al., 2004; Meyer et al., 2004).

Mammalian cell culture studies suggested that Nlgs play a role in synapse formation (Scheiffele et al., 2000; Dean et al., 2003; Chih et al., 2004; Nam and Chen, 2005). However, in vivo knockout studies of mouse Nlgs and Nrxs revealed normal synapse structure and numbers but defective synaptic transmission pointing to their role in synapse function (Missler et al., 2003; Varoqueaux et al., 2006), as opposed to synapse formation. To further analyze the Nlg/Nrx function, recent studies used Dro- 
sophila to circumvent the functional redundancy issues and address the function of these proteins in vivo (Li et al., 2007; Zeng et al., 2007; Banovic et al., 2010).

Genome analyses in Drosophila identify four Nlg-like proteins (CG31146, CG13772, CG34127, and CG34139) (Biswas et al., 2008; Banovic et al., 2010; Sun et al., 2011). We have been attempting to determine the role of CG13772 [Drosophila Neuroligin 2 (Dnlg2)], but during the final stages of preparation of this work, Sun et al., (2011) reported the characterization of a null mutation in $d n \lg 2$. Here we report the generation of an independent null allele of $d n \lg 2$. We show that loss of Dnlg2 results in reduced synaptic development and neurotransmission. The synaptic function of Dnlg2 is only restored when Dnlg2 is expressed both presynaptically and postsynaptically at the neuromuscular junctions (NMJs), unlike what was reported (Sun et al., 2011). Furthermore, postsynaptic overexpression of Dnlg2 causes reduction in bouton growth, whereas combined presynaptic and postsynaptic overexpression leads to synaptic bouton overgrowth. We show that double mutants of $d n r x$ (Li et al., 2007) and $d n l g 2$ are fully viable and display phenotypes that resemble $d n l g 2$ and $d n r x$ single mutants. We therefore reach different conclusions than Sun et al. (2011). Our results reveal that Dnlg2 is required presynaptically and postsynaptically for synapse development and function at NMJs, and that both proteins largely affect the same biological processes in vivo, i.e., determining the proper number of active zones (AZs) and the size of the presynaptic densities.

\section{Materials and Methods}

Cloning of dnlg2 full-length cDNA. A PCR fragment was amplified from fly genomic DNA based on sequence homology with the vertebrate Neuroligin-1. The PCR fragment was radiolabeled to screen a Drosophila 0-20 h embryonic cDNA library. Overlapping partial cDNA clones were isolated, sequenced, and compiled as into a full-length cDNA sequence of 4195 bps encoding an open reading frame of 1248 aa. This cDNA corresponds to $d n \lg 2$. The GenBank accession number of $d n \lg 2$ sequence is AAF52450.

In situ hybridization. PCR-amplified DNA fragments from the 3 'region of $d n \lg 2$ cDNA were amplified and labeled with digoxigenin-UTP (Roche) as sense and antisense probes and used for in situ hybridization following standard protocols (Kearney et al., 2004).

Production and purification of Dnlg2 Antibody. Guinea pig polyclonal antibodies against Dnlg2 were generated using a recombinant protein containing the cytoplasmic region of Dnlg2 fused with glutathione $S$-transferase (GST) at the N terminus (GST-Dnlg2-CT). The serum was affinity purified after passing it through a GST-Sepharose column followed by binding with GST-Dnlg2-CT-Sepharose. The purified antibody was used at a dilution of 1:50 for immunostaining and 1:100 for immunoblot analysis.

Generation of dnlg2 mutants. dnlg2-null alleles were generated by targeted deletion using FLP-FRT recombination (Parks et al., 2004; Thibault et al., 2004). A $P$ insertion upstream of the $d n \lg 2$ genomic locus, $P\{X P\} d 02251$, and a piggyBac insertion downstream of dnlg2 locus, $P B a c\{W H\} f 04579$, were selected. The males from $P\{X P\} d 02251$ and $P B a c\{W H\} f 04579$ were individually crossed to virgin females bearing FLP recombinase. Male progeny carrying both $P\{X P\} d 02251$ and FLP recombinase were crossed to females carrying $P B a c\{W H\} f 04579$ and FLP recombinase. After 2 days of egg laying, the parents and progeny were both heat shocked at $37^{\circ} \mathrm{C}$ for $1 \mathrm{~h}$. On the third day, the parents were removed and the progeny were heat shocked for $1 \mathrm{~h}$ each day for 4 more consecutive days. After eclosion, mosaic virgin females were mated with $y w ; L / C y O$ males. The red-eye progeny males were individually crossed to $y w ; L / C y O$ virgin females to obtain balanced stocks which were analyzed for $d n l g 2$ deletion by PCR. The following primers were used to verify the targeted deletion of $d n \lg 2$ locus and to determine the breakpoints of the deletion: 5'-TGCTGAGCGCAACAAGGACCA-3', 5'-CGGGTGAATC TCTCCCACTAA-3', 5' -CCAAAGCTCCCGGATTTACC-3', 5' -CTAC
GTAAAGACTCGGCCCCATTCAGC-3'， 5' -CTAACATCTCATCTGG GTCCTC-3' ' 5' -GACCAGGAGATCAAGATCCGC-3' ' 5' -CCGAGTCC AAGTCCAACTACA- $3^{\prime}$, and $5^{\prime}$-CGGTTTTGGAATTCTCTAGAAATC TCTTTA-3'

A dnlg2-null allele was outcrossed to an isogenized $w^{1118}$ Canton-S line (a gift from V. Budnik) for seven generations and two independent lines $d n \lg { }^{C L 2}$ and $d n \lg 2^{C L 5}$ were balanced over a green fluorescent protein (GFP) balancer. For each set of experiment, homozygous $d n l g 2 / d n l g 2$ non-GFP wandering third-instar larvae were collected for experimental analyses.

Fly stocks and genetics. The same isogenized $w^{1118}$ line used for outcrossing $d n l g 2$-null allele served as the control for all analyses. $P$ [acman] BAC CH322-173I20 (Venken et al., 2009), which carries the entire dnlg2 genomic locus, was used to generate transgenic flies using PhiC31 integrase-mediated site-specific transgenesis (attP docking site at 68A4) (Bateman et al., 2006). The UAS-dnlg2 flies used in rescue experiments were provided by G. Boulianne (Sun et al., 2011). The dnrx-null allele, $d n r x^{273}$, was used for the genetic analyses in this study (Li et al., 2007). $D f(3 R) 5 C 1$ (referred in the text as $D f$ ), which uncovers the $d n r x$ locus, has been described previously (Li et al., 2007). Gal4 lines used for Dnlg2 overexpression were as follows: C57-Gal4 (Budnik et al., 1996) and 24BGal4 (Luo et al., 1994) (expressed mainly in the musculature), elav-Gal4 (expressed in all neurons) (Lin and Goodman, 1994), and $t u b^{P}$-Gal4 (expressed ubiquitously) (Lee and Luo, 1999). All stocks and crosses were raised at $21^{\circ} \mathrm{C}$. For each set of experiments, all genotypes and crosses were transferred to fresh culture at the same time to maintain consistency. Other fly stocks were obtained from the Drosophila Stock Center (Bloomington, IN).

Immunostaining, confocal microscopy, and bouton number quantification. Preparation and antibody staining for whole-mount embryos and dissected wandering third-instar larvae were performed as described previously (Li et al., 2007). Dissected larval fillets were fixed in Bouin's fixative for $15 \mathrm{~min}$. The following antibodies were used: guinea pig antiDnlg2 (1:50, this study), guinea pig anti-Dnrx (1:500; Li et al., 2007), mouse anti-GluRIIA (1:250), rabbit anti-GluRIII (1:2000; Marrus et al., 2004), rabbit anti-Dlg (1:2000; Woods and Bryant, 1991), and mouse monoclonal anti-Brp (1:500; Wagh et al., 2006). The Dnrx signal at the NMJ was detected by using the VECTASTAIN ABC system (Vector Laboratories) and Tyramide Signal Amplification (Invitrogen-Invitrogen) (Li et al., 2007). Secondary antibodies conjugated to Alexa 488, 568, and 647 (Invitrogen-Invitrogen) were used at 1:400. Fluorescence-conjugated antihorse radish peroxidase (HRP) (Jackson ImmunoResearch Laboratories) antibodies were used at 1:50.

Samples for each set of experiments were processed simultaneously, stained in the same tube, and imaged with the same parameters using Olympus FV1000 confocal microscope. Quantification of bouton numbers was performed at muscles $6 / 7$ and muscle 4 of abdominal segment 3 . Type Ib boutons at NMJ6/7 and at NMJ4 were visualized and quantified by staining of body wall muscle preparations with anti-HRP and antiDlg. Quantification for bouton numbers was normalized to wild type.

Quantification of fluorescence intensity. Control $w^{1118}$ and all mutants were immunostained with anti-GluRIIA and anti-Brp or anti-GluRIII and anti-Brp. Terminal boutons at each branch of NMJ6/7 from five to seven animals of each genotype were scanned by confocal microscopy. Confocal stacks were acquired using the same settings with $0.25 \mu \mathrm{m}$ steps through the entire synaptic boutons. Images were processed using Volocity 5.3 (Improvision) software. The fluorescent intensity of GluRIIA or GluRIII in each terminal bouton was determined by integrating the fluorescent intensity of the areas with 15 to $100 \%$ intensity of the whole image. The integrated intensity of GluRIIA or GluRIII was then divided by the number of AZs in each bouton to obtain the level of GluRIIA or GluRIII fluorescence intensity per AZ. The total number of AZs at NMJ6/7 was quantified using the function "separate touching objects" of Volocity. The areas with anti-Brp staining intensity at 10 to $100 \%$ were selected and the touching dots were separated using $0.03 \mu \mathrm{m}$ as the size reference.

Electron microscopy and morphometric analysis. For ultrastructural NMJ studies, third-instar larval fillets were dissected at room temperature in ice-cold, calcium-free HL-3 medium (Stewart et al., 1994) con- 
taining $70 \mathrm{~mm} \mathrm{NaCl}, 5 \mathrm{~mm} \mathrm{KCl}, 20 \mathrm{~mm} \mathrm{MgCl}$, $10 \mathrm{~mm} \mathrm{NaHCO} 3,5 \mathrm{~mm}$ trehalose, $5 \mathrm{~mm}$ HEPES, and $115 \mathrm{~mm}$ sucrose, $\mathrm{pH}$ 7.2, and subsequently fixed overnight in $4 \%$ paraformaldehyde/ $1 \%$ glutaraldehyde $/ 0.1 \mathrm{M}$ cacodylic acid, pH 7.2. Microwave irradiation with the PELCO BioWave 34700 laboratory microwave system was used for subsequent electron microscopy (EM) processing steps. After overnight fixation, the fixed fillets were additionally fixed at $640 \mathrm{~W}$ with a cycle of $10 \mathrm{~s}$ on, $20 \mathrm{~s}$ off, $10 \mathrm{~s}$ on, followed by $4 \times$ water rinses at $150 \mathrm{~W}$ for $40 \mathrm{~s}$ each, postfixed with $1 \%$ aqueous osmium tetroxide $2 \times$ at $90 \mathrm{~W}$ with a cycle of $2 \mathrm{~min}$ on, $2 \mathrm{~min}$ off, 2 min on under vacuum. Then they were placed on ice in between changes with additional $1 \mathrm{~h}$ incubation on rotator, dehydrated in increasing ethanol concentrations $1 \times$ at $150 \mathrm{~W}$ for $40 \mathrm{~s}$ each, followed by propylene oxide $2 \times$ at $250 \mathrm{~W}$ for $40 \mathrm{~s}$ each. Samples were gradually infiltrated with increasing resin to propylene oxide ratio up to full resin $2 \times$ at $250 \mathrm{~W}$ for $3 \mathrm{~min}$ each under vacuum. The samples were embedded in flat silicone mold with EMBED-812 resin and cured in the oven at $60^{\circ} \mathrm{C}$.

ImageJ $1.40 \mathrm{~g}$ (National Institutes of Health, USA) was used for morphometric analyses of EM images. Only Ib boutons (diameter $>1.5 \mu \mathrm{m}$ ) with clear subsynaptic reticulum (SSR) from muscles 6 and 7 in the third and fourth segments were examined and quantified. The bouton diameter was determined by bouton perimeter divided by $\pi(\sim 3.141593)$.

SSR width was quantified as described in Budnik et al. (1996). Three to four different measurements were made from postsynaptic density (PSD) to distal SSR for each bouton. The SSR width was then calculated by averaging these measurements. To reduce the effect of bouton size, the averaged SSR width was further normalized by the diameter of the bouton (averaged SSR width/bouton diameter). The postsynaptic area was defined as the area between the PSD and the SSR. Only those AZs that clearly showed postsynaptic area were measured. (N represents the number of boutons analyzed while $n$ is the number of AZs).

Electrophysiology. Wandering third-instar larvae were dissected in icecold zero calcium HL-3 solution (Stewart et al., 1994). Dissected larvae were then rinsed three times with HL-3 with $0.5 \mathrm{~mm} \mathrm{Ca}^{2+}$ and incubated in HL-3 with $0.5 \mathrm{~mm} \mathrm{Ca}^{2+}$ for at least 3 min before recording. Body wall muscle 6 (abdominal segment A3 only) was used for intracellular recordings with sharp electrodes filled with a 2:1 mixture of $2 \mathrm{~m}$ potassium acetate and $2 \mathrm{~m}$ potassium chloride (32-40 M $\Omega$ ). Data were collected only when resting membrane potential was below $-65 \mathrm{mV}$. The recording data were discarded when resting membrane potential shifted more than $\pm 5 \mathrm{mV}$ during the course of experiment. In addition, only one muscle per larvae was recorded in each individual experiment. Excitatory junction potentials (EJPs) were recorded by directly stimulating the segmental nerve innervating each hemisegment $\mathrm{A} 3$ through a glass capillary electrode (internal diameter, $\sim 10 \mu \mathrm{m}$ ) at $0.2 \mathrm{~Hz}$. The applied currents were $6 \pm 3 \mu \mathrm{A}$ with fixed stimulus duration at $0.3 \mathrm{~ms}$. This is $50 \%$ more than that required to activate both $\mathrm{Ib}$ and Is boutons on the recorded muscles. Twenty to thirty evoked EJPs were recorded for each muscle for analysis. Miniature EJP (mEJP) events were collected for $5 \mathrm{~min}$. Both EJPs and mEJPs were amplified with an Axonclamp 2B amplifier in bridge mode under the control of Clampex 8.2 (Molecular Devices). All experiments were performed at room temperature $\left(20^{\circ} \mathrm{C}-22^{\circ} \mathrm{C}\right)$.

EJPs and paired-pulse stimulation were analyzed with pClamp 9.2 software (Molecular Devices). mEJPs was analyzed using the Mini Analysis Program (Synaptosoft). Evoked EJP amplitude was corrected by using nonlinear summation (Feeney et al., 1998). The quantal content of evoked release was calculated from individual muscles by ratio of the averaged EJP and averaged mEJP amplitude. Statistical analyses of EJP and mEJPs between genotypes were made using Student's $t$ test (SigmaPlot 10.0, Systat Software).

Immunoprecipitation and Immunoblotting Analysis. The immunoprecipitation (IP) experiments were performed as previously described (Banerjee et al., 2010). Briefly, fly heads of desired genotypes were homogenized using a glass homogenizer in a weight/volume ratio of 1:3 in ice-cold lysis buffer containing $50 \mathrm{~mm}$ HEPES, pH 7.2), $100 \mathrm{~mm} \mathrm{NaCl}$, $1 \mathrm{~mm} \mathrm{MgCl} 2,1 \mathrm{~mm} \mathrm{CaCl}_{2}$, and 1\% NP-40 with protease inhibitors. The lysates were kept on ice for $10 \mathrm{~min}$ and centrifuged at $50,000 \times \mathrm{g}$ for 30 $\min$ at $4^{\circ} \mathrm{C}$, and used subsequently for IP and immunoblot analysis. For each IP reaction, $100 \mu \mathrm{l}$ of supernatant was precleared with Protein A beads followed by incubation with primary antibodies at 1:20 dilution (anti-Dnlg2, anti-Dnrx) for $8 \mathrm{~h}$ at $4^{\circ} \mathrm{C}$. The supernatant-antibody mix was incubated with $25 \mu \mathrm{l}$ of prewashed Protein A beads for $2 \mathrm{~h}$ at $4^{\circ} \mathrm{C}$. The beads were then washed three times in PBS followed by elution of the immunocomplexes in $30 \mu \mathrm{l}$ of PBS/SDS buffer and resolved by SDSPAGE for immunoblotting with respective antibodies. Anti-Dnlg2 was used at 1:100 and anti-Dnrx was used at 1:500 for immunoblot analysis.

\section{Results}

\section{Generation of dnlg2-null mutants}

The domain structure of Dnlg2 is similar to that of mammalian Nlg1. The extracellular domain contains an N-terminal signal peptide and an AChE-like domain. This is followed by a transmembrane domain (TM) and a cytoplasmic region with a PDZ binding motif (PBM) (Fig. 1A). The AChE domains of Drosophila Dnlg2 and human Nlg1 (NCBI Reference Sequence: NP_055747.1) (Saus et al., 2010) share $\sim 36 \%$ amino acid sequence identity and $\sim 56 \%$ similarity (Fig. 1A). To determine the dnlg2 expression in the Drosophila we performed in situ hybridization in embryos. A dnlg2 probe recognizing the TM region revealed that $d n \lg 2$ is primarily expressed in the ventral nerve cord (VNC) and the brain of stages 14-16 embryos (Fig. $1 B$ ). In addition, Dnlg2 expression is also observed at low levels in the embryonic musculature (data not shown).

To study the function of Dnlg2 in vivo, we generated dnlg2null mutants using a P-element and a PiggyBac that flank the gene and carry FRTs. Upon induction of FLP in the germline the DNA between the FRT sites is deleted (Parks et al., 2004; Thibault et al., 2004) (Fig. 1C) resulting in a $32.6 \mathrm{~kb}$ deletion that includes the dnlg2 locus and CG13773 (Fig. 1C). To avoid issues with the genetic background, two $d n l g 2$ deletion alleles were outcrossed to an isogenized wild-type strain for seven generations and two independent excision stocks named $d n \lg 2^{C L 2}$ and $d n \lg 2^{C L 5}$ were established. Both $d n \lg 2^{C L 2}$ and $d n \lg 2^{C L 5}$ are homozygous viable. The endpoints of the deletions were established by PCR using the primers shown (Fig. $1 D$ ).

Next we generated antibodies against Dnlg2 to determine its subcellular localization in the third-instar larvae. Immunostaining using anti-Dnlg2 and anti-Bruchpilot (Brp), a marker for presynaptic AZs (Wagh et al., 2006; Weyhersmüller et al., 2011) indicates that Dnlg2 and Brp are localized to CNS synapses of the VNC (Fig. $1 E$ ). No staining was observed in $d n l g 2$ mutants (Fig. $1 F)$. To determine whether Dnlg2 is present presynaptically and/or postsynaptically at larval NMJs, we performed immunostaining of third-instar larval musculature. Despite generating 11 antibodies against Dnlg2, we were unable to detect Dnlg2 at NMJs. Although Sun et al. (2011) reported that Dnlg2 localizes postsynaptically at the larval NMJs, we were not able to detect NMJ labeling using the anti-Dnlg2 with the protocol reported by Sun et al. (2011). We thus conclude that Dnlg2 levels at the larval NMJs are too low to be consistently detected.

To determine the relative molecular weight of Dnlg2 and to establish that $d n \lg 2^{C L 2}$ is indeed a null allele, we performed immunoblot analysis of wild-type and $d n \lg 2^{C L 2}$ adult head lysates using a polyclonal guinea pig anti-Dnlg2 antibodies (this study). As shown in Figure $1 G$, wild-type lysates show the presence of a robust $\sim 70 \mathrm{kDa}$ band (Fig. $1 G$, a, red arrowhead) at a shorter exposure time, which is absent in the $\mathrm{dnlg}^{\mathrm{CL2}}$ lysates. Upon longer exposure (30 min) a faint $\sim 145 \mathrm{kDa}$ band (Fig. $1 G$, b, red arrow, asterisk) is visible, which is absent from $d n l g 2$ lysates. To confirm the presence of the $\sim 145 \mathrm{kDa}$ band in the wild-type lysates, we split the blot and probed them separately with antiDnlg2 (Fig. $1 H$ ). We were able to detect both $\sim 145 \mathrm{kDa}$ (Fig. $1 \mathrm{H}$, 

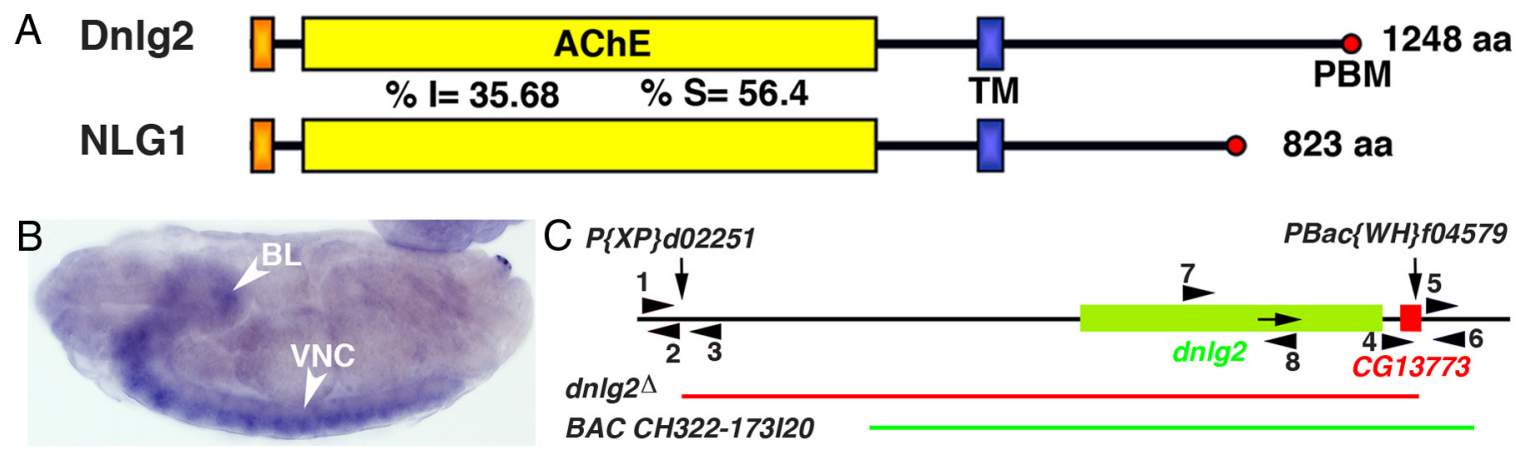

BAC CH322-173120
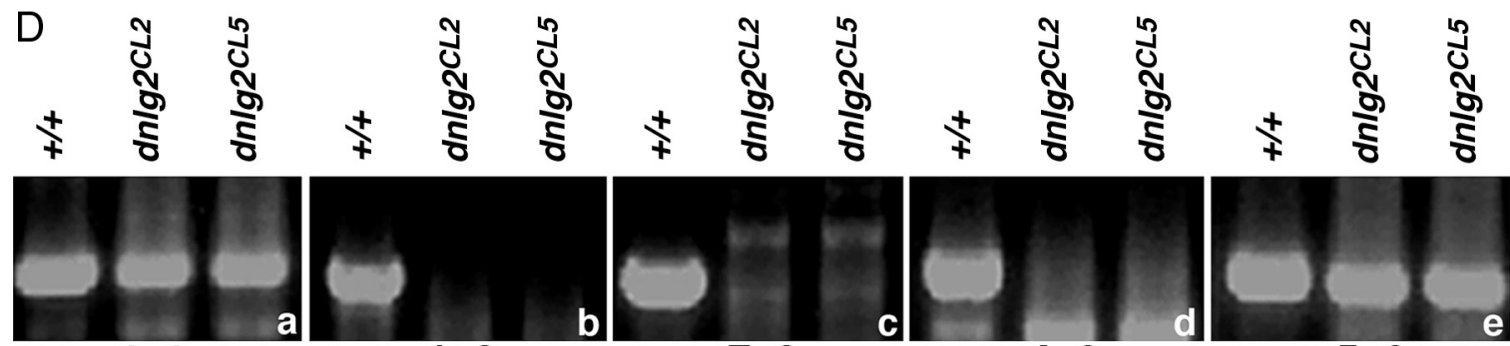

$1+2$

$1+3$

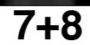

$4+6$

$5+6$

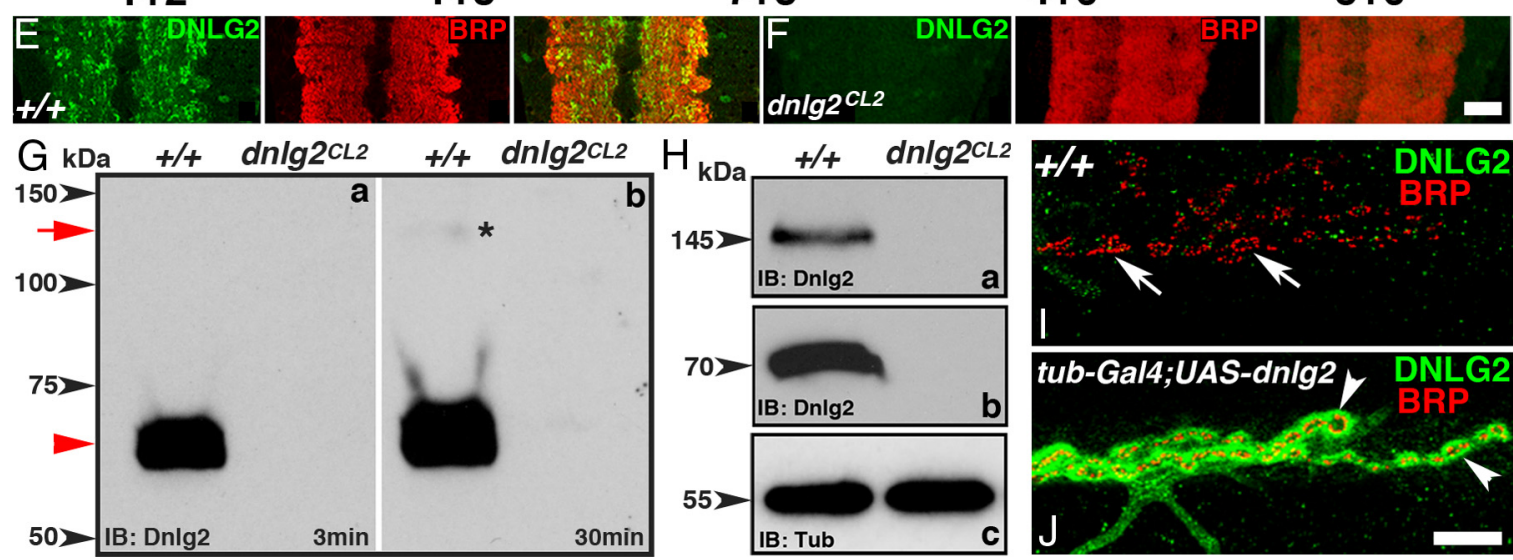

Figure 1. Generation of dnlg2 mutants. A, Protein domain structure of Drosophila Dnlg2 and human NLG1. Similar to human NLG1, Dnlg2 is composed of a signal peptide, an AChE-like domain and a TM domain followed by a PBM at the C terminus. The percentage amino acid identity (I) and similarity (S) between Dnlg2 and NLG1 in the AChE domains are indicated. $\boldsymbol{B}$, In situ hybridization of wild-type embryo at stage 16 using a dnlg2-labeled antisense probe shows mRNA expression in the VNC (arrowhead) and brain lobes (BL, arrowhead). C, Genomic structure of dnlg2 and the flanking insertions, $P\{X P\} d 02251$ in the $5^{\prime}$ end and PBac\{WH\}f04579 in the $3^{\prime}$ end. The arrows pointing down indicate the sites of insertion. The arrow in the dnlg2 locus shows the direction of transcription. The dnlg2-null mutant was generated using FRT-based recombination. The deleted genomic region is shown by the red line. A genomic $B A C$ construct, $P[a c m a n] B A C C H 322-173120$, spanning the region shown by the green line was used to rescue the deletion. $\boldsymbol{D}, P C R$ confirmation of the targeted deletion using different primer combinations. The PCR primer sets used are shown as numbers and arrowheads in $\boldsymbol{C}, \boldsymbol{E}, \boldsymbol{F}, \mathrm{VNC}$ sections from third-instar larvae of wild-type $(\boldsymbol{E})$ and $d n \lg 2^{C L 2}$ mutants $(\boldsymbol{F})$ stained with anti-Dnlg2 (green) and anti-Brp (red) show expression of Dnlg2 in the synapse-rich regions of the VNC where Brp is expressed ( $\boldsymbol{E}$, merged image). Dnlg2 expression is absent in dnlg2 mutants $(\boldsymbol{F}) . \boldsymbol{G}, \boldsymbol{H}$, Immunoblot analysis of Dnlg2. Adult fly head extracts from wild-type $(+/+)$ and $d n l g 2$ mutants immunoblotted with anti-Dnlg2 antibodies. A shorter $(G$, a) and a longer $(G, b)$ exposure time reveal the presence of a strong $\sim 70 \mathrm{kDa}$ band in the wild-type lysate (red arrowhead). The blot with the longer exposure time shows the appearance of a faint $\sim 145 \mathrm{kDa}$ band $(G, \mathrm{~b}$, red arrow, asterisk). Immunoblots with anti-Dnlg2 antibodies processed separately $(H, a, b)$ detects the upper $\sim 145 \mathrm{kDa}$ molecular weight $(H$, a, arrowhead) and the bottom $\sim 70 \mathrm{kDa}$ band $(H, \mathrm{~b}$, arrowhead) in wild-type lysates that are absent in the $d n l g 2$ lysates. For protein-loading control, the blot was probed with anti- $\alpha$-tubulin $\left(H, c\right.$, arrowhead). $I, J$, Third-instar larval NMJ from wild-type $(I)$ and tub ${ }^{P}$-Gal $4 / U A S$-dnlg2 $(J)$ show expression of Dnlg2 (green) and Brp (red) at the NMJ synaptic boutons. Scale bars: (in $\boldsymbol{F}$ ) $\boldsymbol{E}, \boldsymbol{F}, 20 \mu \mathrm{m}$; (in $\boldsymbol{J}) \boldsymbol{I}, \boldsymbol{J}, 10 \mu \mathrm{m}$.

a) and $\sim 70 \mathrm{kDa}($ Fig. $1 \mathrm{H}$, b) bands in wild-type lysates that were missing from the $d n \lg 2$ lysates using this process. We conclude that the $145 \mathrm{kDa}$ band is specific to Dnlg2 and is only visualized when immunoblots are processed separately from the $70 \mathrm{kDa}$ band, most likely, as the levels of the $70 \mathrm{kDa}$ band are many folds higher than that of the $145 \mathrm{kDa}$ band. The $145 \mathrm{kDa}$ molecular weight of Dnlg2 is slightly higher than that predicted from the open reading frame $(\sim 138 \mathrm{kDa})$ and was not observed by Sun et al. (2011). These data show that $d n \lg 2$ is indeed a null allele.

Since our immunohistochemical analysis could not detect the presence of Dnlg2 at the wild-type larval NMJ (Fig. 1I, arrows), we overexpressed the full-length $U A S$ - $d n \lg 2$ ubiquitously using $t u b^{P}$-Gal4 driver (Fig. $1 \mathrm{~J}$ ). Upon staining with anti-Brp (red) and
anti-Dnlg2 (green), we were able to detect Dnlg2 at the NMJ synaptic boutons (Fig. $1 \mathrm{~J}$ ). In summary, our data indicate that Dnlg2 is a $145 \mathrm{kDa}$ protein and that it may undergo proteolytic processing or degradation to form a $70 \mathrm{kDa}$ isoform. It can easily be detected in the synaptic-rich areas of the larval VNC, but its abundance at NMJs is probably very low.

dnlg2 mutants exhibit a reduced number of boutons at larval NMJs

To determine whether the NMJs were affected we performed immunostaining on the larval body walls of wild-type and $d n \lg 2$ mutants using anti-HRP to identify neuronal membranes and anti-Dlg to label type I boutons (Fig. 2A) (Budnik et al., 1990; 

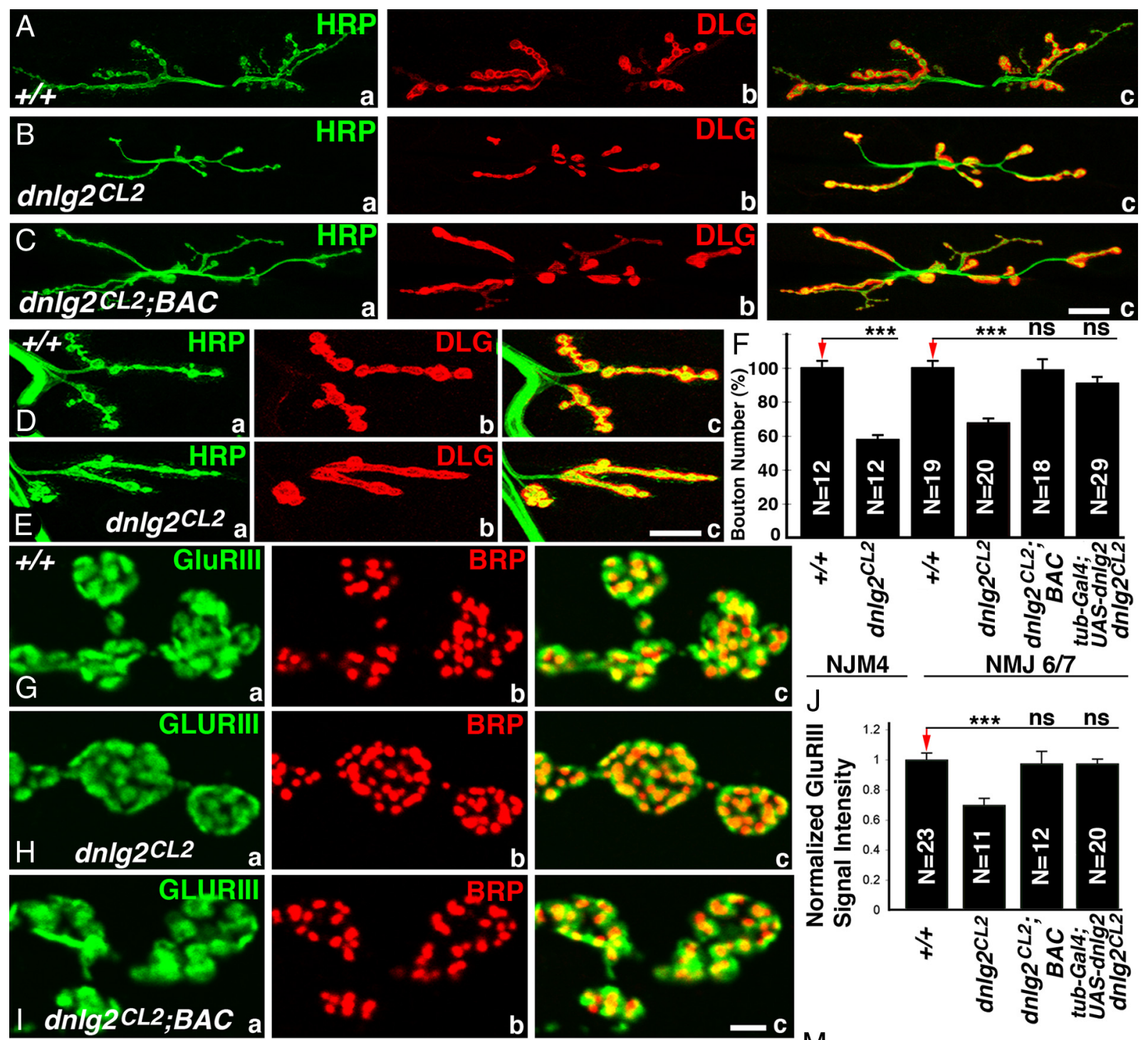

\section{NJM4}

NMJ 6/7
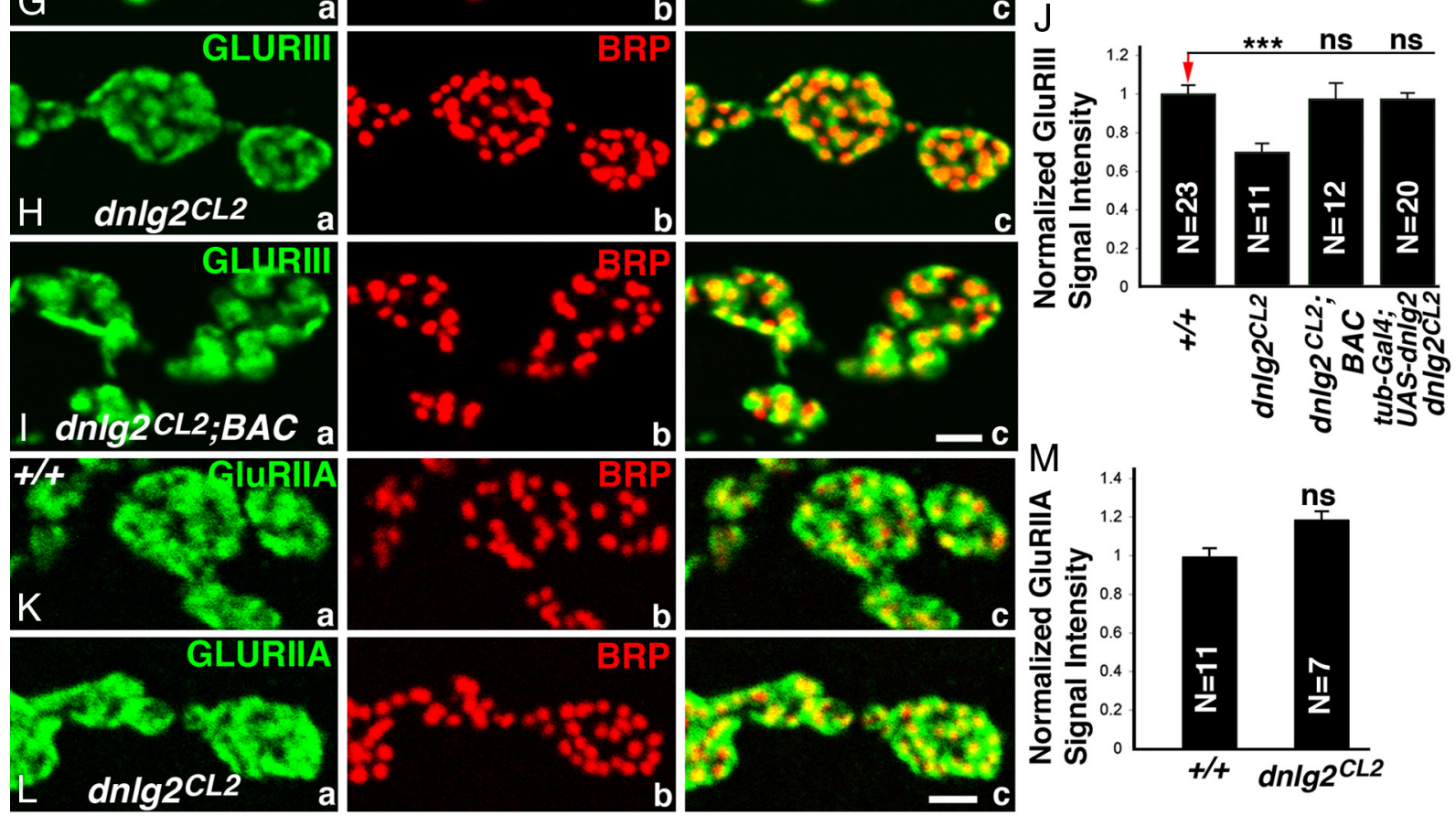

Figure 2. Synaptic bouton growth at NMJs is reduced in dnlg2 mutants. $A-E$, Confocal images of NMJ6/7 $(A-C)$ and NMJ4 $(D, E)$ from abdominal segment 3 of third-instar larvae labeled with anti-HRP (green) and anti-Dlg (red). Compared with wild-type NMJ6/7 ( $\boldsymbol{A})$, dnlg2 homozygous mutants $(\boldsymbol{B})$ show reduced NMJ expansion and fewer boutons. This phenotype is rescued by a $B A C$ transgene containing dnlg2 genomic region ( $(\boldsymbol{C}$. AtNMJ 4, compared with wild type (D), dnlg2 homozygous mutants $(\boldsymbol{E})$ have fewer boutons, which appeared to be merged. $\boldsymbol{F}$, Quantifications oftype lb and ls bouton number at NMJ6/7 and type Ib bouton number at NMJ4 adjusted to wild-type bouton number. The bouton number deficits in $\mathrm{dnl} l \mathrm{2}$ mutants are rescued by $B A C$ transgene or by ubiquitous Dnlg2 expression using tub ${ }^{P}-G a l 4$. $G-I$, Confocal images of synaptic boutons at segment 3 NMJ6/7 labeled with postsynaptic marker, GluRIII (green) and AZ marker, Brp (red). The alignment of presynaptic and postsynaptic areas appears to be unaffected in $d n l g 2$ mutants $(H, c)$. However, the levels of GluRIIl in dnlg2 mutants $(H$, a $)$ are significantly reduced. This phenotype is rescued by the BAC transgene $(I$, a $) . J$, Quantification of GluRlll signal intensity with $3 \mathrm{D}$ reconstructed confocal images using Volocity software also reveals reduction in intensity in $\mathrm{dn}_{\mathbf{g}} 2^{(12}$ mutants. $\boldsymbol{K}, \boldsymbol{L}$, Confocal images of synaptic boutons at NMJ6/7 labeled with postsynaptic marker, GluRIIA (green) and BRP (red). The alignment of GluRIIIA with AZ and the levels of GluRIIA are unaffected in dnlg2 mutants ( $L$ ). $M$, Quantification of GluRllA signal intensity shows slight but not significant increase in dnlg2 mutants compared with wild type. Error bar indicates SEM:****p $<0.001 ;{ }^{* *} p<0.01 ;{ }^{*} p<0.05$ (Student's $t$ test). Scale bars: $A-E, 20 \mu \mathrm{m} ; \mathbf{G}-\boldsymbol{L}, 2 \mu \mathrm{m}$.

Lahey et al., 1994). As shown in Figure 2 B, a-c, in $d n l g 2$ mutants, the number of boutons is severely reduced: they have fewer boutons at muscle 6/7 (NMJ6/7) (Fig. $2 \mathrm{~B}, \mathrm{a}-\mathrm{c}$ ) and muscle 4 (NMJ4) (Fig. $2 E, \mathrm{a}-\mathrm{c}$ ) when compared with wild type (Fig. $2 A, D$; quan- tified in Fig. 2 F). This defect is caused by the loss of $d n \lg 2$ and/or $C G 13773$ as this phenotype as well as other phenotypes (see below) are rescued with a genomic $B A C(P[$ acman $] B A C C H 322-$ 173I20; indicated by the green line in Fig. $1 D$; Venken et al., 2009) 

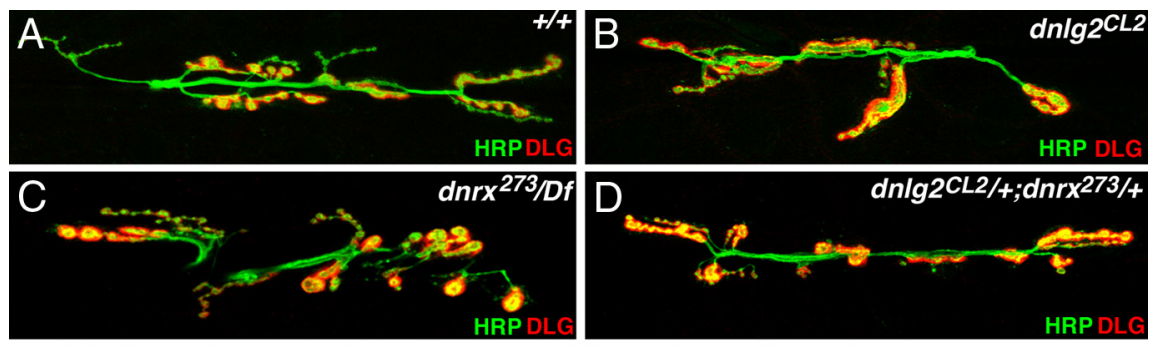

dnlg2CL2/+;dnrx273/t
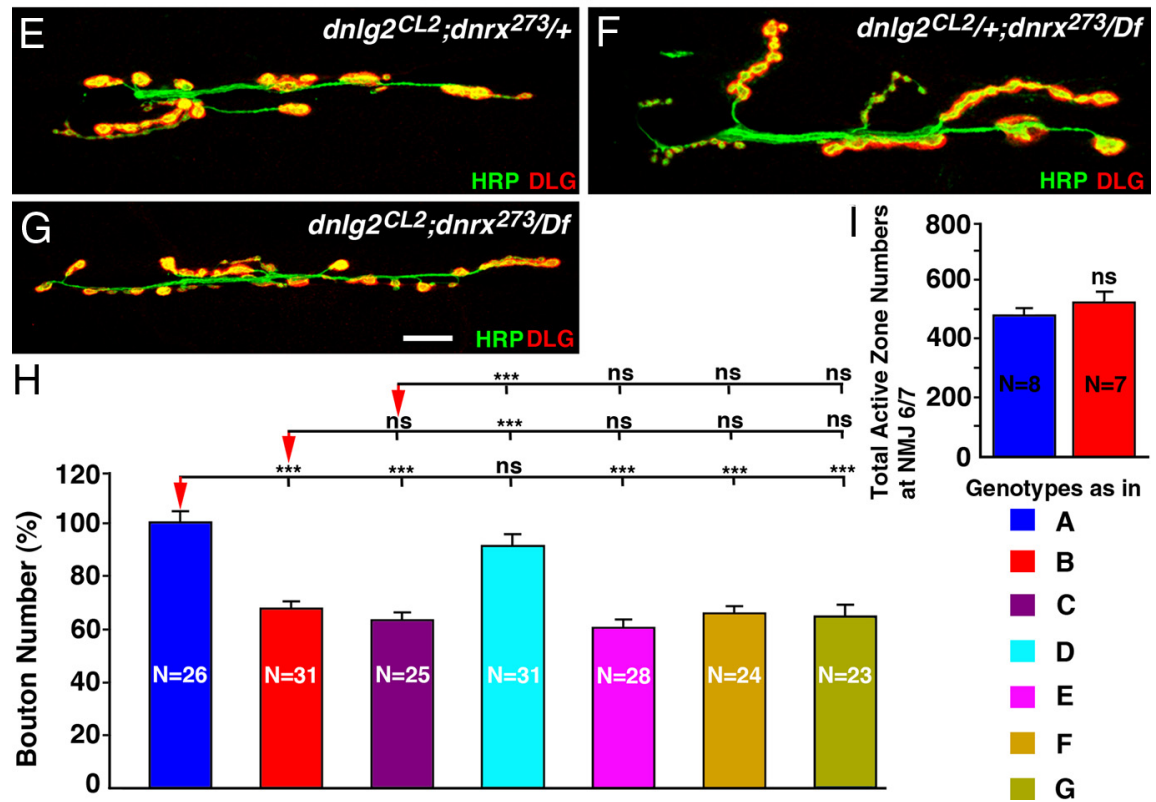

Figure 3. $\quad d n l g 2$ and $d n r x$ mutants display similar NMJ developmental defects. $\boldsymbol{A}-\boldsymbol{G}$, Confocal images of NMJ6/7 from abdominal segment 3 in third-instar larvae labeled with anti-HRP (green) and anti-DLG (red). Compared with control $\left(w^{1118}\right)(\boldsymbol{A}), d n l g 2$ mutants $(\boldsymbol{B}), d n r x / D f$ mutants $(\boldsymbol{C}), d n l g 2 ; d n r x^{+\prime-}(\boldsymbol{E}), d n l g 2^{+\prime-} ; d n r x(\boldsymbol{F})$, and $d n l g 2 ; d n r x$ double homozygous $(\boldsymbol{G})$ show fewer boutons. The NMJ morphology of transheterozygous $d n \lg 2^{+/-} ; d n r x^{+/-}(\boldsymbol{D})$ is unaffected. $\boldsymbol{H}$, Quantification of total bouton number at NMJ6/7 adjusted to control bouton number. $d n \lg 2 ; d n r x / D f$ single mutants, $d n \lg 2 ; d n r x^{+/-}, d n l g 2^{+/-} ; d n r x / D f$, and $d n l g 2$; dnrx/Df double homozygous have $\sim 60-62 \%$ boutons compared with control. ${ }^{* * *} p<0.001 ;{ }^{* *} p<0.01 ;{ }^{*} p<0.05$ (ANOVA, Tukey's post hoc test). Scale bar, $20 \mu \mathrm{m}$. I, Quantification of total AZ numbers at NMJ6/7. dnlg2 mutants have a comparable number of AZs with those observed at the wild-type NMJs (Student's $t$ test). Genotypes in $\boldsymbol{H}$ are color coded and are same as in $\mathbf{A}-\mathbf{G}$.

that contains the entire genomic region of $d n \lg 2$ and $C G 13773$ (Fig. 2C,F). However, CG13773 is not implicated as ubiquitous expression of UAS-Dnlg2 using $t u b^{P}$-Gal4 driver restores bouton number to wild-type levels in the $d n \lg 2$ excision mutants (Fig. 2 F; see below). The boutons in dnlg2 mutants (Fig. $1 E$ ) are less defined when compared with the wild type (Fig. 1C). The wild-type synaptic boutons have a rounded to oval morphology and are separated from each other by a distinct neural process giving a beaded appearance (Fig. $2 E$, a) whereas the $d n \lg 2$ mutant boutons are not well separated (Fig. 2 E, b). These data show that loss of Dnlg2 causes a reduction of boutons as well as an aberrant overall morphology.

To examine the distribution and localization of presynaptic and postsynaptic proteins at the $d n l g 2$ mutant synapses, we performed immunostaining using anti-Brp (presynaptic) and antiGluRIII (postsynaptic), which labels one of the subunits of Drosophila glutamate receptors (Marrus et al., 2004). Although all AZs have both Brp and GluRIII punctae juxtaposed to each other (Fig. 2G-I), the level of GluRIII is reduced in $d n l g 2$ mutants (Fig. $2 \mathrm{H}$, a) compared with wild type (Fig. $2 \mathrm{G}$, a). Quantification of the fluorescent intensity of GluRIII punctae suggests that there is a $30 \%$ decrease in $d n l g 2$ mutants (Fig. $2 J$ ). This phenotype is also rescued by genomic $B A C$ construct or by ubiquitous Dnlg2 overexpression using $t u b^{P}$-Gal4 driver in dnlg2 mutants (Fig.
2I,J; data not shown). However, staining with anti-Brp and anti-GluRIIA, another subunit of glutamate receptors, showed that there is a slight, but not statistically significant, increase in the level of GluRIIA in $d n \lg 2$ mutants (Fig. $2 \mathrm{~K}-\mathrm{M}$ ). These studies suggest that Dnlg2 is required for proper synaptic development and proper postsynaptic protein assembly at the NMJs.

$d n \lg 2$ and $d n r x$ affect NMJ morphology and function in a similar manner

Studies in mice have indicated that Nrxs and Nlgs interact in trans to function at the synapse (Südhof, 2008). Moreover, Banovic et al. (2010) recently presented data that presynaptic DNRX affects Dnlg1 clusters in the postsynaptic densities. However, it has been argued that $d n r x$ and $d n l g 2$ serve different functions at the NMJ as double mutants have a much more severe reduction in bouton number than either of the single mutants (Sun et al., 2011). To assess whether Dnlg2 and Dnrx serve similar or different functions at the NMJ synapses, we examined the morphology and the bouton numbers at the larval NMJs of $d n \lg 2$ and $d n r x$ single and double mutants. Both $d n \lg 2$ (Fig. 3B) and $d n r x / D f$ (Fig. $3 C$ ) single mutants are null mutations that display a significantly reduced number of boutons compared with their wild-type counterpart (Fig. $3 A, H$ ). Larvae transheterozygotes for $d n \lg 2^{+/-}$; $d n r x^{+/-}$exhibit normal NMJ morphology (Fig. $3 D, H$ ) similar to the wild type (Fig. $3 A, H)$. However, $d n \lg 2^{-/-}$; $d n r x^{+/-}$(Fig. 3E,H), dnlg2 $2^{+/-} ; d n r x / D f$ (Fig. $3 F, H$ ), and $d n \lg 2^{-/-} ; d n r x / D f($ Fig. $3 G, H$ ) all display a similar reduction in bouton numbers as $d n \lg 2$ (Fig. $3 B, H$ ) and $d n r x / D f$ single mutants (Fig. 3C,H). The differences in bouton numbers between these mutant genotypes (Fig. $3 B-G$ ) do not reach any statistical significance. Furthermore, the total AZ numbers as visualized by anti-Brp staining at NMJ6/7 did not show any significant difference between the wild-type and $d n \lg 2 \mathrm{mu}-$ tants (Fig. 3I). In addition, whereas Sun et al. (2011) documented that $d n \lg 2^{-1-} ; d n r x^{-1-}$ are lethal, we find that our double-null mutants are viable, further suggesting that loss of $d n l g 2$ and/or $d n r x$ does not exacerbate the phenotype of the other, consistent with the conclusion that both proteins affect the same molecular events and cause very similar phenotypes at the NMJs.

\section{dnlg2 mutants exhibit synaptic differentiation defects at the NMJs}

The Drosophila larval NMJ synapse displays stereotypic ultrastructure including the presynaptic T-bars and densities as well as postsynaptic specializations, the SSR (Zhai and Bellen, 2004; Fouquet et al., 2009). Since dnlg2 mutants display synaptic growth defects at the NMJs (Fig. 2), we examined the ultrastructural features associated with the loss of Dnlg2 at synapses. We performed transmission electron microscopy (TEM) analyses on dnlg2 mutants. Cross sections of the wild-type boutons show 
several AZs with characteristic T-bars surrounded by synaptic vesicles (Fig. 4A) (Mendoza-Topaz et al., 2008; Fouquet et al., 2009). A wild-type synapse at a higher magnification shows an AZ, the postsynaptic area (PSA), and SSR (Fig. 4B). These NMJ synaptic boutons are embedded in the muscle and surrounded by specialized membrane folds, the SSR. Several defects were observed in $d n \lg 2$ mutants. $d n \lg 2$ mutant boutons exhibit an increased number of AZs in each bouton (Fig. 4C). Interestingly, the space between postsynaptic density and the SSR, the PSA, is increased in dnlg2 mutants (Fig. 4C,D; quantified in Fig. $4 J$ ). In addition, we find that the width of SSR is severely reduced in dnlg2 mutants. All these phenotype are rescued by introduction of a $B A C$ construct $(P[$ acman $] B A C C H 322-173 I 20)$ that contains the genomic region of $d n l g 2$ (Fig. $4 E$; quantified in Fig. $4 H-K$ ).

The increase in AZ number per bouton is also observed in $d n r x$ mutants (Fig. 4F) (Li et al., 2007) and double mutants of $d n \lg 2$ and $d n r x$ also exhibit a similar increased AZ numbers and defective PSAs (Fig. 4G). Consistent with an increase in number of AZs, we observed an increase in length of postsynaptic density per unit perimeter in all mutants. Together, these data indicate that Dnlg2 plays a critical role in proper postsynaptic differentiation and that Dnlg2 and Dnrx are jointly required for proper synapse organization and maturation.

\section{Dnlg2 and Dnrx form a molecular complex}

The morphological analyses presented in the preceding sections indicate that Dnlg2 and Dnrx function together to coordinate synaptic growth at the NMJs. To test whether Dnlg2 and Dnrx are present in the same molecular complex, we performed IP followed by immunoblot analyses using anti-Dnlg2 and anti-Dnrx antibodies. When anti-Dnlg2 antibodies were used for IP in wildtype adult fly head extracts, we were able to IP the $145 \mathrm{kDa}$ Dnlg2 protein (Fig. 5A). When anti-Dnrx antibodies were used for IP in adult wild-type and $d n \lg 2$ fly heads, the anti-Dnlg2 antibody detected the $145 \mathrm{kDa}$ Dnlg2 protein in the IP complex (Fig. 5B) of wild type but not $d n \lg 2$. Interestingly, in the same blot, the $70 \mathrm{kDa}$ Dnlg2 could not be detected in both the wild-type and $d n \lg 2$ IP complex (Fig. 5C, arrowhead). These results show that Dnlg2 $(145 \mathrm{kDa})$ and Dnrx are present in the same molecular complex. When fly head lysates from wild-type and $d n l g 2$ mutants were immunoprecipitated using anti-Dnlg2 antibodies, the Dnlg2 (70 $\mathrm{kDa}$ ) was abundantly detected in the wild type but not in the dnlg2 mutants (Fig. 5D). To further determine whether loss of Dnlg2 had any effect on the protein stability and levels of Dnrx, we performed immunoblot analysis of equal amounts of wildtype and $d n \lg 2$ mutant adult head lysates. We found that the levels of Dnrx in $d n \lg 2$ mutants are comparable to those in the wild type, suggesting that the stability of Dnrx is not affected in $d n \lg 2$ mutants (Fig. 5E). Same amounts of lysates from wild-type and dnlg2 fly heads immunoprecipitated with anti-Dnrx antibodies showed the presence of Dnrx in both wild-type and $d n \lg 2 \mathrm{mu}-$ tants (Fig. 5F). These data indicate that the full-length $145 \mathrm{kDa}$ Dnlg2 is most likely the functional protein present in the Dnrx complex, while the $70 \mathrm{kDa}$ Dnlg2 might be a processed form that is not present in the Dnrx molecular complex.

\section{Dnlg2 is required presynaptically and postsynaptically for synaptic development at NMJs}

Vertebrate studies have shown that Nlgs that are expressed postsynaptically interact with Nrxs expressed exclusively presynaptically (Song et al., 1999; Graf et al., 2004; Chih et al., 2005; Nam and Chen, 2005; Südhof, 2008; Wittenmayer et al., 2009). These conclusions were challenged as Nrxs were also observed to be expressed postsynaptically pointing to a complex mechanism of interactions between Nrxs and Nlgs in synapse function and modulation (Peng et al., 2004; Taniguchi et al., 2007). To establish whether Dnlg2 function is required presynaptically and/or postsynaptically at NMJs, we performed rescue analyses of $d n \lg 2$ mutants (Fig. $6 B, C$ ) by driving $U A S$ - $d n \lg 2$ presynaptically (neurons), postsynaptically (muscle), or ubiquitously (Fig. $6 D-N$ ). When Dnlg2 was expressed postsynaptically using musclespecific drivers, C57-Gal4 and 24B-Gal4, the reduction in bouton number at $d n \lg 2$ mutant $\mathrm{NMJ}$ s could not be rescued in $d n \lg 2^{C L 2}$ (Fig. 6D-G; quantified in Fig. 6O). Similarly, expression of Dnlg2 presynaptically using neuronal driver, elav-Gal4, also failed to rescue $d n \lg 2 \mathrm{NMJ}$ phenotypes (Fig. $6 \mathrm{H}$; quantified in Fig. 6O). However, when Dnlg2 was expressed in both neurons and muscles with a ubiquitous driver, $t u b^{P}$-Gal4, the bouton number was restored to wild-type levels in $d n l g 2^{C L 2}$ mutants (Fig. 6I, J; quantified in Fig. 6O). These data show that Dnlg2 is required presynaptically and postsynaptically for proper bouton formation and growth at NMJs. We further confirmed that the ability to rescue the $d n l g 2$ phenotype using tubP-Gal4 compared with $24 B-G a l 4$ or C57-Gal4 is not due to higher expression of Dnlg2 in muscles. Interestingly, fluorescence signal intensity quantification showed that Dnlg2 expression in muscles under $24 B-G a l 4$ is significantly higher than $t u b^{P}$-Gal4 while C57-Gal4 is statistically comparable to that of C57-Gal4 (data not shown). Sun et al. (2011) previously reported that Dnlg2 functions postsynaptically and that $d n \lg 2$ mutant phenotypes at the NMJs are fully rescued by postsynaptic expression of Dnlg2. However, we failed to rescue their $d n \lg 2^{K O 70}$ mutants (Sun et al., 2011) by postsynaptic expression of Dnlg2 using 24B-Gal4 and C57-Gal4 (Fig. 6E, G,O). Together, our data indicate that presynaptic or postsynaptic expression alone of Dnlg2 is not sufficient to rescue $d n l g 2$ mutant NMJ phenotypes; rather Dnlg2 is required presynaptically and postsynaptically for proper bouton formation.

Several vertebrate studies have shown that overexpression of Nlgs is sufficient to promote synapse formation in cultured mammalian neurons (Scheiffele et al., 2000; Comoletti et al., 2003; Prange et al., 2004). We therefore assessed whether overexpression of Dnlg2 in the wild-type animals affected normal bouton growth at NMJs. Surprisingly, postsynaptic overexpression of Dnlg2 using (C57-Gal4 and 24B-Gal4) reduced bouton numbers to levels similar to those observed in $d n \lg 2$ mutants (Fig. $6 \mathrm{~K}, \mathrm{~L}$; quantified in Fig. 6P). However, presynaptic overexpression of Dnlg2 using elav-Gal4 had no effect on bouton growth (Fig. 6M; quantified in Fig. $6 P$ ). In contrast, when Dnlg2 was overexpressed both presynaptically and postsynaptically using $t u b^{P}$ Gal4, we observe an increase in bouton growth of $\sim 27 \%$ when compared with wild type (Fig. $6 \mathrm{~N}$; quantified in Fig. 6O). Hence, Dnlg2 promotes bouton formation and synaptic growth at NMJs when expressed presynaptically and postsynaptically during development.

\section{Synaptic transmission is reduced in $\mathbf{d n l g} 2$ mutants}

As shown in the preceding sections, loss of Dnlg2 results in the reduction of GluRIII levels at NMJs (Fig. $2 J$ ) and ultrastructural abnormalities at the synapse (Fig. 4). We next examined the consequences of loss of Dnlg2 alone as well as the combined loss of Dnlg2 and Dnrx on synaptic transmission at the NMJs. We performed our electrophysiological analyses on muscle 6 of thirdinstar larval body walls and recorded the EJPs in $0.5 \mathrm{mM}\left[\mathrm{Ca}^{2+}\right]_{0}$ at $0.2 \mathrm{~Hz}$. Both $d n \lg 2^{C L 2}$ and $d n \lg 2^{K O 70}$ mutants exhibit a reduction in EJP amplitude, which is rescued by the genomic $B A C$ construct in $d n \lg 2^{C L 2}$ (Fig. $7 A$ ). Under identical conditions, $d n r x$ 

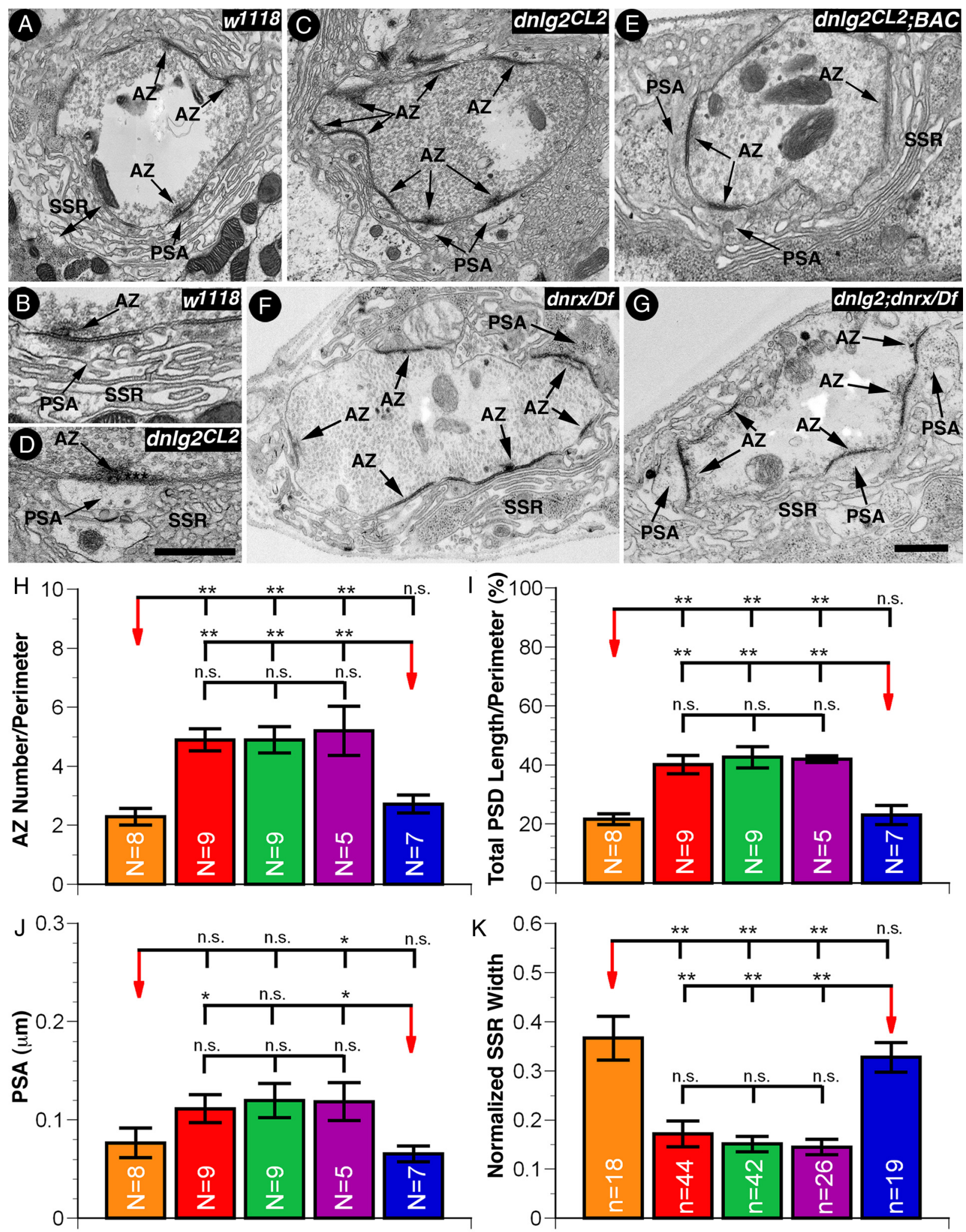

dnlg2;BAC $\square d n l g 2 \square d n r x / D f \quad \square d n l g 2 ; d n r x / D f \quad \square w 1118$

Figure 4. dnlg2 mutants display synapse differentiation defects with severely disorganized postsynaptic areas. $\boldsymbol{A}-\mathbf{G}$, TEM micrographs of wild type $(\boldsymbol{A}, \boldsymbol{B})$, dnlg2 mutants $(\boldsymbol{C}, \boldsymbol{D})$, and $d n l g 2$ mutants with the genomic $B A C$ construct $(\boldsymbol{E}), d n r x / D f(\boldsymbol{F})$, and $d n l g 2 ; d n r x / D f$ double mutants $(\boldsymbol{G})$ showing the ultrastructural features of boutons at NMJ 6/7. AZ, PSAs, and SSR are highlighted. SSR widths were measured from PSD to the distal fold. Note that the number of AZs (arrows) is increased and PSAs fail to differentiate properly in $d n l g 2$ mutants (C, D, PSA with arrows). $\boldsymbol{H}-\boldsymbol{K}$, Quantification of ultrastructural morphometric analyses on all genotypes. $\boldsymbol{H}$, Compared with wild type, the AZ number in boutons is increased in $d n l g 2^{C L 2}$ mutants. $I$, The lengths of PSD adjusted to the perimeter in $d n l g 2$ mutants is also increased. PSAs are enlarged $(\boldsymbol{J})$ and the widths of SSR are reduced $(\boldsymbol{K})$ in $d n l g 2$ mutants. ${ }^{* * *} p<0.001 ;{ }^{* *} p<0.01 ;{ }^{*} p<0.05$ (Student's $t$ test). Scale bars: $0.5 \mu \mathrm{m}$. 


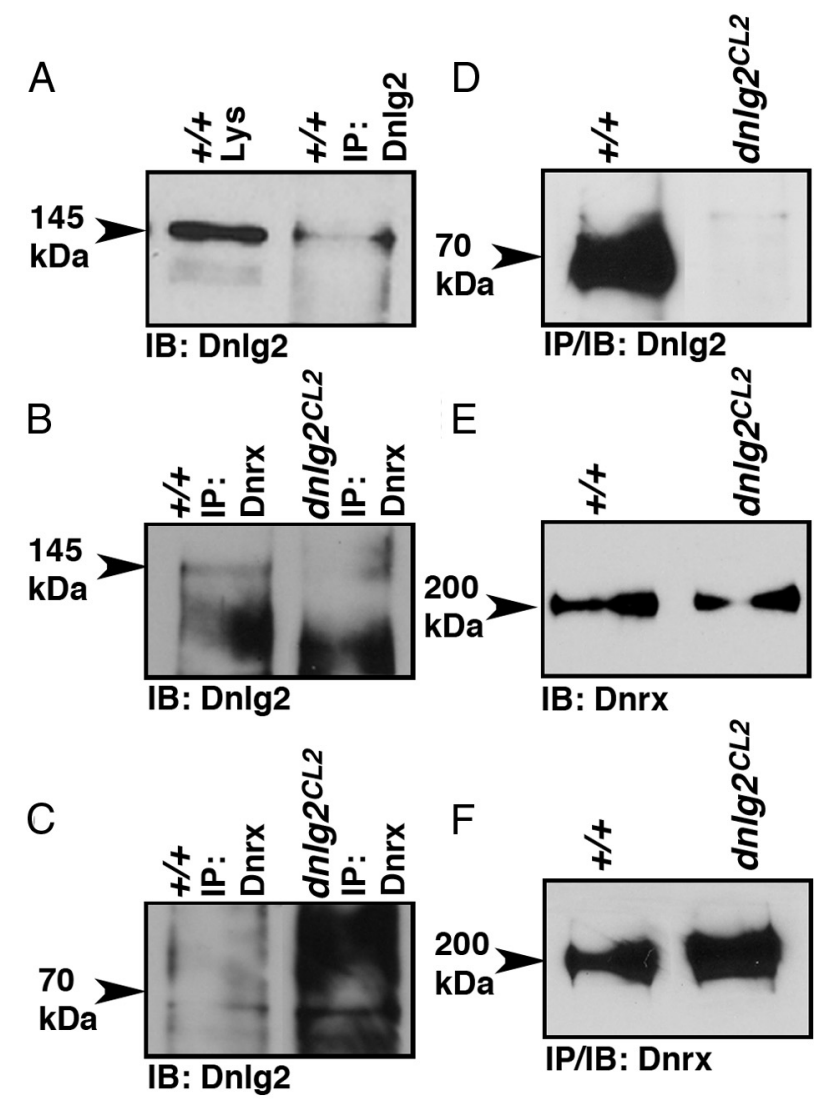

Figure 5. Dnlg2 forms a biochemical complex with Dnrx. A, IPs from wild-type fly head lysates immunoblotted (IB) using anti-Dnlg2 antibodies show the presence of Dnlg2 (145 kDa, arrowhead). $B, C, I P$ from wild-type fly head lysates using anti-Dnrx reveals the presence of Dnlg2 in the same complex (B, $145 \mathrm{kDa}$, arrowhead). The $70 \mathrm{kDa}$ Dnlg2 does not associate with Dnrx. Only nonspecific background bands are observed in the wild-type and $d n l g 2$ lysates near where the $70 \mathrm{kDa}$ band is expected ( $\boldsymbol{C}$, arrowhead). Note that $\boldsymbol{B}$ and $\boldsymbol{C}$ are from the same protein blot probed separately. $\boldsymbol{D}$, IPs from equal amounts of wild-type and $d n l g 2$ mutant fly head lysates using anti-Dnlg2 show the presence of Dnlg2 $(70 \mathrm{kDa})$ in wild-type but not in $d n / g 2$ mutants. (Note that the break in between the lanes is due to removal of an empty lane). $\boldsymbol{E}$, Dnrx protein levels are unaffected in $d n$ lg 2 mutants. $F$, IPs from equal amounts of wild-type and dnlg2 mutants using anti-Dnrx show the presence of Dnrx in both wild-type and $d n l g 2$ mutants.

mutants also have reduced EJP amplitudes, consistent with previous reports (Zeng et al., 2007; Chen et al., 2010). Interestingly, $d n \lg 2$; dnrx double mutants show a similar reduction in EJP amplitudes as $d n \lg 2$ or $d n r x$ single mutants, again suggesting that Dnlg2 and Dnrx function together at the synapse. We observed no significant changes in $\mathrm{mEJP}$ amplitudes in all mutant combinations when compared with control wild type (data not shown) and $d n \lg 2^{C L 2}$;BAC-Res (Fig. $7 B$ ). All mutant combinations revealed severely decreased quantal contents compared with wild type (data not shown) and the genomic $B A C$ rescue of $d n \lg 2$ mutants (Fig. 7C). Interestingly, the total number of AZs at the NMJs on muscle 6/7 are comparable between wild-type and $d n \lg 2$ mutants (Fig. 3I), indicating that $d n \lg 2$ mutants have a lower release probability due to synaptic structural alterations.

Next we determined the EJP, mEJP amplitudes, and the quantal contents when Dnlg2 was expressed using neuronal, muscle, and ubiquitous drivers in $d n \lg 2^{C L 2}$ and $d n \lg 2^{\text {KO7O }}$ mutant backgrounds. Both dnlg2 mutants showed a significant reduction in EJP amplitudes (Fig. 7A) and quantal content (Fig. 7C) when Dnlg2 was expressed either presynaptically or postsynaptically. The EJP amplitude, however, was similar to control levels $\left(d n \lg 2^{C L 2} ; B A C-\right.$ Res $)$ in both dnlg2 mutants when Dnlg2 was ex- pressed both presynaptically and postsynaptically using $t u b^{P}$. Gal4 (Fig. 7A). The mEJP amplitudes in $d n \lg 2^{C L 2}$ mutants were not significantly different upon Dnlg2 expression presynaptically and/or postsynaptically when compared with $d n \lg 2^{C L 2} ; B A C$-Res (Fig. $7 B$ ). However, in $d n \lg 2^{K O 70}$ mutants, postsynaptic expression of Dnlg2 resulted in a significant increase in the mEJP amplitude (Fig. 7B) when compared with wild-type and mutant larvae expressing Dnlg2 presynaptically or ubiquitously. Together our data show that Dnlg2 is required both presynaptically and postsynaptically for proper synaptic transmission.

\section{Discussion}

Sequence analyses of the Drosophila genome revealed four neuroligin genes and mutational analyses of two of these genes $d n \lg 1$ (Banovic et al., 2010) and $d n \lg 2$ (Sun et al., 2011) revealed that Dnlg1 and Dnlg2 are required independently for synaptic growth and function. Dnlg1 functions postsynaptically and is required for proper synaptic development and differentiation (Banovic et al., 2010). Dnlg2 was also shown to function postsynaptically (Sun et al., 2011); however, some of the previously reported functions of Dnlg2 are inconsistent with the data presented here. We report the generation of mutations in $d n \lg 2$ and characterization of the associated phenotypes. We find that loss of $d n \lg 2$ causes a developmental defect at NMJs, with reduced bouton numbers. This phenotype is fully rescued when $d n l g 2$ was expressed presynaptically and postsynaptically, indicating that Dnlg2 is required in both presynaptic and postsynaptic compartments for normal synaptic growth. Ultrastructural analyses revealed that $d n \lg 2$ mutants have significantly increased numbers of AZs and postsynaptic density length. However, the postsynaptic SSR width is reduced. Electrophysiological measurements revealed that $d n \lg 2$ mutants have reduced EJP amplitude, but normal mEJP amplitude, indicating a reduced release probability. Furthermore, $d n l g 2$ and $d n r x$ double mutants are viable and reveal phenotypes similar to $d n l g 2$ and $d n r x$ single mutants, indicating that $d n l g 2$ and $d n r x$ likely function in the same pathway to coordinate synaptic development and transmission. Finally, our phenotypic rescue data using the Gal4/UAS system (Brand and Perrimon, 1993) suggest that Dnlg2 is required both presynaptically and postsynaptically for proper NMJ bouton growth, synapse structure, and neurotransmission.

Although some of our results are in agreement with published data on $d n \lg 2$, many of the results reported here are in disagreement with the data presented in Sun et al. (2011). First, it was reported that postsynaptic Dnlg2 expression alone is sufficient to rescue the $d n \lg 2$ mutant phenotypes. Using the $d n \lg 2$ mutant alleles reported in Sun et al. (2011) and postsynaptic Dnlg2 expression, we were unable to rescue the bouton growth phenotypes. Second, it was reported that EJP amplitudes are much increased in dnlg2 mutants. However, we find that EJP amplitudes in both $d n \lg 2$ and $d n \lg 2^{K O 70}$ mutants are decreased and that both mutants exhibit a reduction in neurotransmitter release probability. Third, our biochemical studies support the existence of a $\sim 145 \mathrm{kDa}$ molecular weight Dnlg2 that based on protein interaction data are most likely the functional form. This form was not reported in Sun et al. (2011). Fourth and perhaps most importantly, $d n \lg 2$ and $d n r x$ double mutants were reported to be lethal by Sun et al. (2011) and to display a more severe phenotype in bouton growth phenotype than individual mutants, suggesting that $d n \lg 2$ and $d n r x$ function in parallel pathways to affect the same biological process. We find that the double mutants are viable and have defects that resemble the $d n \lg 2$ and $d n r x$ singlenull mutants in overall NMJ morphology and at the ultrastructural level, strongly indicating that they do not function in 

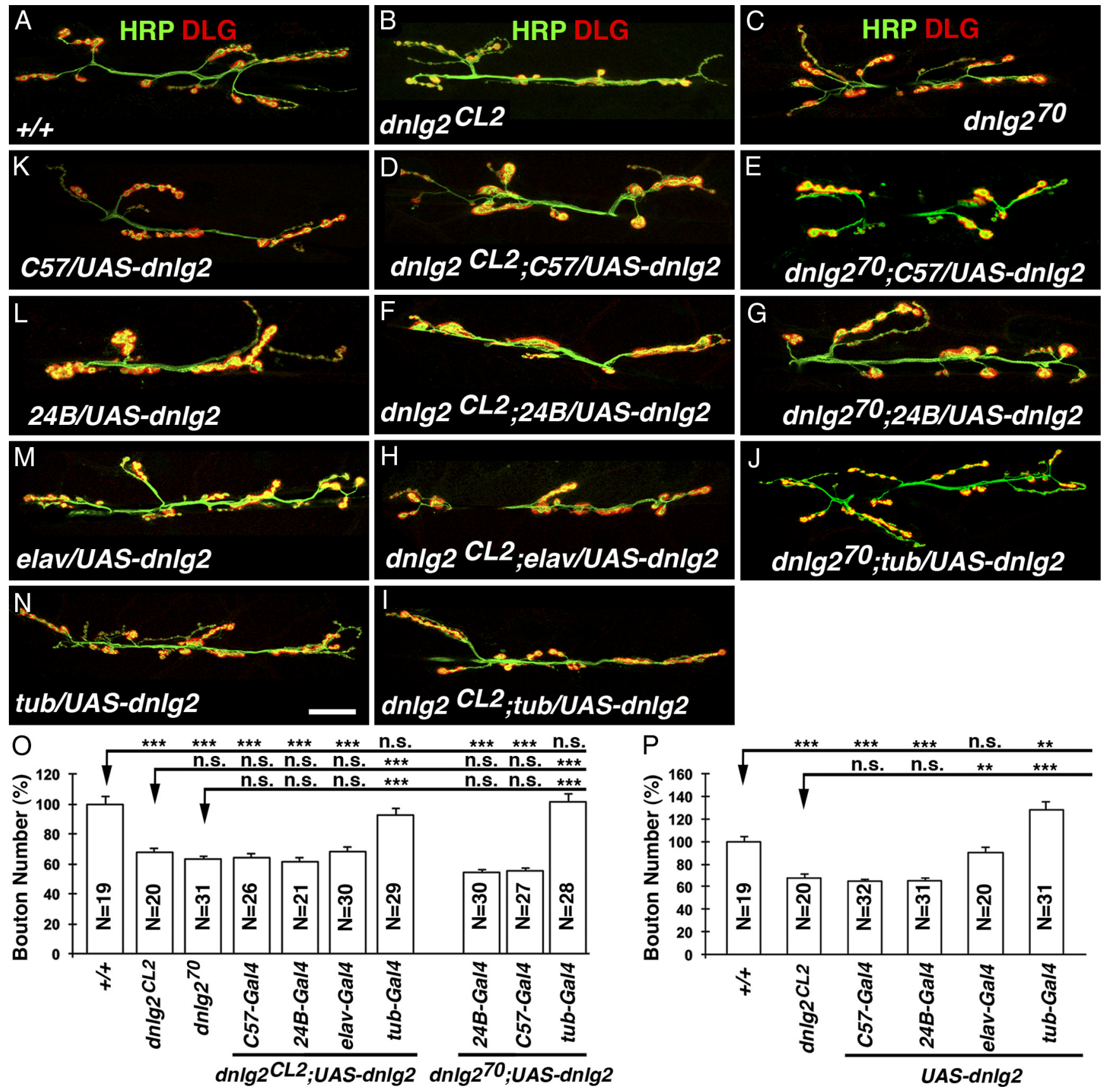

Figure 6. Dnlg2 is required presynaptically and postsynaptically for proper synaptic growth at NMJs. $A-J, d n l g 2$ CDNA transgene rescue analyses at NMJ 6/7. Compared with two dnlg2 mutants, $d n l g 2^{C L 2}(\boldsymbol{B})$ and $d n \lg 2^{K 070}\left(\boldsymbol{C} ;\right.$ Sun et al., 2011), expression of Dnlg2 in muscles with C57-Gal4 $(\boldsymbol{D}, \boldsymbol{E})$ or $24 B$-Gal4 $(\boldsymbol{F}, \boldsymbol{G})$ failed to rescue bouton number deficits in both $d n l g 2^{C L 2}$ and $d n l g 2^{K 070}$ mutants. Similarly, expression of Dnlg2 in neurons using elav-Gal4 $(\boldsymbol{H})$ also failed to rescue the NMJ phenotype. However, when Dnlg2 was expressed ubiquitously with tub-Gal $4(J, I)$, the NMJ phenotype in both $d n l g 2^{C L 2}$ and $d n l g 2^{K O 70}$ mutants was restored to wild-type levels. $\boldsymbol{K}-\boldsymbol{N}$, dnlg2 overexpression analyses in the wild-type background. Overexpression of Dnlg2 in muscles using $(57-G a l 4(\boldsymbol{K})$ or 24B-Gal4 $(\boldsymbol{L})$ adversely affected bouton growth. In contrast, overexpression of Dnlg2 in neurons $(\boldsymbol{M})$ does not affect NMJ bouton growth or number dramatically. Simultaneous overexpression of Dnlg2 in muscles and neurons promotes bouton growth at NMJs $(\boldsymbol{N}) . \mathbf{O}, \boldsymbol{P}$, Quantification of bouton number at NMJ6/7 for rescue analyses $(\boldsymbol{O})$ and overexpression analyses $(\boldsymbol{P})$. Error bar indicates SEM; ${ }^{* * *} p<0.001 ;{ }^{* *} p<0.01 ;{ }^{*} p<0.05$ (ANOVA, Tukey's post hoc test). Scale bar, $20 \mu \mathrm{m}$.

parallel pathways. A possible explanation of the double mutant phenotypes documented by Sun et al. (2011) is that genetic background issues contribute to the lethality when combined with dnrx mutants. The potential effects of genetic background on NMJ morphology and function have been reported previously in a screen for synaptogenesis mutants (Liebl et al., 2006). Our phenotypic analyses revealed identical results from both $d n l g 2$ alleles [dnlg2 ${ }^{C L 2}$ (this study) and $d n \lg 2^{K O 70}$ (Sun et al., 2011)] ruling out any major contributions from the genetic backgrounds between the two independently generated $d n \lg 2$ alleles. However, the lethality observed in $d n \lg 2 / d n r x$ double mutants reported in Sun et al. (2011) could be attributed to contributions from the genetic background.

Drosophila Nlgs and their role at the synapse

The functions of vertebrate Nrxs and Nlgs are thought to be important for synapse maturation and activity-dependent synaptic modulation, but dispensable for initiation of synaptogenesis 
(Missler et al., 1998, 2003; Südhof, 2008). Several recent studies support these functional roles for Nrxs and Nlgs in synaptic plasticity (Kim et al., 2008; Etherton et al., 2009; Blundell et al., 2010; Dahlhaus et al., 2010; Choi et al., 2011). These studies have raised interesting questions as to whether Drosophila Nlgs are involved in synaptic plasticity and modulation. Both Dnlg1 and Dnlg2 are similar in structure, but seem to function independently for synaptic development, organization, and function (Banovic et al., 2010; Sun et al., 2011; this study). Although dnlg1-null and dnlg2-null mutants display some similarities in their NMJ phenotypes, including reduced NMJ bouton numbers, reduced postsynaptic SSR thickness and reduced overall synaptic transmission, they also show key differences. dnlg1 mutants have fewer AZs at muscle 6/7 and some mutant boutons are completely devoid of postsynaptic markers, which is not observed in dnlg2 mutants. dnlg1 mutants show mostly postsynaptic defects, but dnlg2 mutants also display presynaptic defects, such as lower release probability, in addition to postsynaptic structural abnormalities. The similarities in mutant phenotypes suggest that they both are involved in bouton growth and SSR stability, and the differences in mutant phenotypes indicate that they have distinct functions in coordinating synaptic development and synapse differentiation. It is possible that Dnlg1 is involved in the recruitment or stabilization of the postsynaptic machinery, whereas Dnlg2 serves to fine-tune and refine synapse organization as is revealed by ultrastructural analysis with an increased number of AZs in the remaining boutons (Fig. 4). In the absence of Dnlg2 and Dnrx AZ number increase and the synaptic areas are significantly increased, suggesting that the mutants fail to prune away ectopic AZs and are unable to refine densities. dnlg1 mutants, on the other hand, lack postsynaptic differentiation at the synapses indicating that Dnlg1 and Dnlg2 perform distinct functions during synapse differentiation (Banovic et al., 2010). Interestingly postsynaptic expression of Dnlg1 and Dnlg2 repress bouton growth, implying that postsynaptic Dnlg1 and Dnlg2 may either interact and interfere with the functions of presynaptic proteins or dilute out functions of a key postsynaptic protein(s), which is involved in normal bouton growth. How a single presynaptic Dnrx protein interacts with postsynaptic Dnlg1 and presynaptic and postsynaptic Dnlg2 to coordinate synaptic development remains unresolved.

\section{Presynaptic and postsynaptic requirements of Nlgs}

Many studies have suggested that Nlgs primarily function as postsynaptic adhesion molecules and interact with presynaptic Nrxs (Song et al., 1999; Scheiffele et al., 2000; Berninghausen et al., 2007). However, there may be exceptions to the postsynaptic localization of Nlgs, as it was recently reported that an Nlg in Caenorhabditis elegans is present at both presynaptic and postsynaptic regions (Feinberg et al., 2008). Along similar lines, it was reported that Nrxs are also expressed in the postsynaptic areas, where they may play a role in controlling synaptogenesis by blocking the functions of postsynaptic Nlgs (Taniguchi et al., 2007). These observations suggest that Nrxs and Nlgs could modulate synapse formation by counteracting each other's functions during synapse formation and/or modulation. Our data provide evidence in support of both a presynaptic and postsynaptic function of Dnlg2 in synapse formation. We show that a full complement of boutons at $d n \lg 2$ mutant NMJs is only restored when Dnlg2 is expressed both presynaptically and postsynaptically. Expression of Dnlg2 only presynaptically or postsynaptically was not sufficient to restore bouton growth. Surprisingly, overexpression of Dnlg2 in the postsynaptic areas in the wild-type animals also leads to a reduction in bouton growth, almost similar to 
dnlg2 mutant levels. However, overexpression of Dnlg2 presynaptically did not result in such phenotypes. On the other hand, when Dnlg2 is expressed both presynaptically and postsynaptically in the wild-type larvae, there is excess bouton growth at NMJs, similar to when Dnrx is overexpressed presynaptically ( $\mathrm{Li}$ et al., 2007). These data suggest that a fine balance of the Dnlg2 protein levels is critical for normal bouton growth. It is possible that high levels of postsynaptic Dnlg2 may lead to an uncontrolled or untimely interaction with presynaptic or postsynaptic proteins, such as Dnrx and DNlg1, respectively, and hinder bouton growth at NMJs, leading to phenotypes that are similar to dnlg2 or dnrx mutants. A recent study also suggested that some $\mathrm{Nlg}$ functions are neurexin-independent and that Nlgs can form complexes with other proteins at the synapses (Ko et al., 2009). This raises the possibility that presynaptic and postsynaptic Dnlg2 functions are dependent on formation of homophilic interactions with itself or heterophilic interactions with other synaptic proteins across the synaptic cleft to organize bouton growth at NMJs. It would be of significant interest to determine how loss of Dnlg2 leads to increased AZs and how mechanistically these functions of Dnlg2 are linked with Dnrx and other synaptic proteins.

In summary, our results show that Neuroligin functions are required presynaptically and postsynaptically for synapse development. Our observations in Drosophila and those of Feinberg et al. (2008) in C. elegans suggest that Nlgs have presynaptic and postsynaptic functions that may be required to counterbalance the functions of Nrxs or other proteins during synaptic growth and modulation. It was recently suggested that postsynaptic Nrxs counter the functions of Nlgs to ensure that synapses do not form at random places. However, in our model, antagonistic functions are unlikely given the similarity in phenotypes between the two mutants. Other synaptic adhesion molecules, such as LRRTM2 (Ko et al., 2009) and the recently identified Teneurins (Mosca et al., 2012) as new interacting partners of Dnrx and Dnlg1, respectively, further add to the complexity of trans-synaptic interactions and synapse organization. In this context, the requirement of Dnlg2 in both the presynaptic and postsynaptic compartments raises interesting questions about how synaptic organization is fine-tuned, and how signaling pathways regulate the expression of presynaptic and postsynaptic proteins during synaptic development and maturation. Deciphering the signaling role of Nrxs and Nlgs at the Drosophila synapses coupled with structure/function analyses should provide a better understanding of the underlying molecular mechanisms of synapse development and function. Such information will provide critical insights into how these molecules are involved in human health and diseases such as ASD.

\section{References}

Banerjee S, Blauth K, Peters K, Rogers SL, Fanning AS, Bhat MA (2010) Drosophila neurexin IV interacts with Roundabout and is required for repulsive midline axon guidance. J Neurosci 30:5653-5667. CrossRef Medline

Banovic D, Khorramshahi O, Owald D, Wichmann C, Riedt T, Fouquet W, Tian R, Sigrist SJ, Aberle H (2010) Drosophila neuroligin 1 promotes growth and postsynaptic differentiation at glutamatergic neuromuscular junctions. Neuron 66:724-738. CrossRef Medline

Bateman JR, Lee AM, Wu CT (2006) Site-specific transformation of Drosophila via phiC31 integrase-mediated cassette exchange. Genetics 173: 769-777. CrossRef Medline

Berninghausen O, Rahman MA, Silva JP, Davletov B, Hopkins C, Ushkaryov YA (2007) Neurexin Ibeta and neuroligin are localized on opposite membranes in mature central synapses. J Neurochem 103:1855-1863. CrossRef Medline
Biswas S, Russell RJ, Jackson CJ, Vidovic M, Ganeshina O, Oakeshott JG, Claudianos C (2008) Bridging the synaptic gap: neuroligins and neurexin I in Apis mellifera. PLoS ONE 3:e3542. CrossRef Medline

Blundell J, Blaiss CA, Etherton MR, Espinosa F, Tabuchi K, Walz C, Bolliger MF, Südhof TC, Powell CM (2010) Neuroligin-1 deletion results in impaired spatial memory and increased repetitive behavior. J Neurosci 30: 2115-2129. CrossRef Medline

Brand AH, Perrimon N (1993) Targeted gene expression as a means of altering cell fates and generating dominant phenotypes. Development 118: 401-415. Medline

Budnik V, Zhong Y, Wu CF (1990) Morphological plasticity of motor axons in Drosophila mutants with altered excitability. J Neurosci 10:3754-3768. Medline

Budnik V, Koh YH, Guan B, Hartmann B, Hough C, Woods D, Gorczyca M (1996) Regulation of synapse structure and function by the Drosophila tumor suppressor gene dlg. Neuron 17:627-640. CrossRef Medline

Chen K, Gracheva EO, Yu SC, Sheng Q, Richmond J, Featherstone DE (2010) Neurexin in embryonic Drosophila neuromuscular junctions. PLoS ONE 5:e11115. CrossRef Medline

Chih B, Afridi SK, Clark L, Scheiffele P (2004) Disorder-associated mutations lead to functional inactivation of neuroligins. Hum Mol Genet 13: 1471-1477.

Chih B, Engelman H, Scheiffele P (2005) Control of excitatory and inhibitory synapse formation by neuroligins. Science 307:1324-1328. CrossRef Medline

Choi YB, Li HL, Kassabov SR, Jin I, Puthanveettil SV, Karl KA, Lu Y, Kim JH, Bailey CH, Kandel ER (2011) Neurexin-neuroligin transsynaptic interaction mediates learning-related synaptic remodeling and long-term facilitation in aplysia. Neuron 70:468-481. CrossRef Medline

Comoletti D, Flynn R, Jennings LL, Chubykin A, Matsumura T, Hasegawa H, Südhof TC, Taylor P (2003) Characterization of the interaction of a recombinant soluble neuroligin-1 with neurexin-1beta. J Biol Chem 278: 50497-50505. CrossRef Medline

Craig AM, Kang Y (2007) Neurexin-neuroligin signaling in synapse development. Curr Opin Neurobiol 17:43-52. CrossRef Medline

Dahlhaus R, Hines RM, Eadie BD, Kannangara TS, Hines DJ, Brown CE, Christie BR, El-Husseini A (2010) Overexpression of the cell adhesion protein neuroligin-1 induces learning deficits and impairs synaptic plasticity by altering the ratio of excitation to inhibition in the hippocampus. Hippocampus 20:305-322. Medline

Dean C, Scholl FG, Choih J, DeMaria S, Berger J, Isacoff E, Scheiffele P (2003) Neurexin mediates the assembly of presynaptic terminals. Nat Neurosci 6:708-716. CrossRef Medline

Etherton MR, Blaiss CA, Powell CM, Südhof TC (2009) Mouse neurexinlalpha deletion causes correlated electrophysiological and behavioral changes consistent with cognitive impairments. Proc Natl Acad Sci U S A 106:17998-18003. CrossRef Medline

Feeney CJ, Karunanithi S, Pearce J, Govind CK, Atwood HL (1998) Motor nerve terminals on abdominal muscles in larval flesh flies, Sarcophaga bullata: comparisons with Drosophila. J Comp Neurol 402:197-209. CrossRef Medline

Feinberg EH, Vanhoven MK, Bendesky A, Wang G, Fetter RD, Shen K, Bargmann CI (2008) GFP reconstitution across synaptic partners (GRASP) defines cell contacts and synapses in living nervous systems. Neuron 57 : 353-363. CrossRef Medline

Fouquet W, Owald D, Wichmann C, Mertel S, Depner H, Dyba M, Hallermann S, Kittel RJ, Eimer S, Sigrist SJ (2009) Maturation of active zone assembly by Drosophila Bruchpilot. J Cell Biol 186:129-145. Medline

Giagtzoglou N, Ly CV, Bellen HJ (2009) Cell adhesion, the backbone of the synapse: "vertebrate" and "invertebrate" perspectives. Cold Spring Harb Perspect Biol 1:a003079. CrossRef Medline

Graf ER, Zhang X, Jin SX, Linhoff MW, Craig AM (2004) Neurexins induce differentiation of GABA and glutamate postsynaptic specializations via neuroligins. Cell 119:1013-1026. CrossRef Medline

Ichtchenko K, Hata Y, Nguyen T, Ullrich B, Missler M, Moomaw C, Südhof TC (1995) Neuroligin 1: a splice site-specific ligand for beta-neurexins. Cell 81:435-443. CrossRef Medline

Iida J, Hirabayashi S, Sato Y, Hata Y (2004) Synaptic scaffolding molecule is involved in the synaptic clustering of neuroligin. Mol Cell Neurosci 27: 497-508. CrossRef Medline

Irie M, Hata Y, Takeuchi M, Ichtchenko K, Toyoda A, Hirao K, Takai Y, 
Rosahl TW, Südhof TC (1997) Binding of neuroligins to PSD-95. Science 277:1511-1515. CrossRef Medline

Jamain S, Quach H, Betancur C, Råstam M, Colineaux C, Gillberg IC, Soderstrom H, Giros B, Leboyer M, Gillberg C, Bourgeron T (2003) Mutations of the X-linked genes encoding neuroligins NLGN3 and NLGN4 are associated with autism. Nat Genet 34:27-29. CrossRef Medline

Kearney JB, Wheeler SR, Estes P, Parente B, Crews ST (2004) Gene expression profiling of the developing Drosophila CNS midline cells. Dev Biol 275:473-492.

Kim J, Jung SY, Lee YK, Park S, Choi JS, Lee CJ, Kim HS, Choi YB, Scheiffele P, Bailey CH, Kandel ER, Kim JH (2008) Neuroligin-1 is required for normal expression of LTP and associative fear memory in the amygdala of adult animals. Proc Natl Acad Sci U S A 105:9087-9092. CrossRef Medline

Ko J, Fuccillo MV, Malenka RC, Südhof TC (2009) LRRTM2 functions as a neurexin ligand in promoting excitatory synapse formation. Neuron 64: 791-798. CrossRef Medline

Lahey T, Gorczyca M, Jia XX, Budnik V (1994) The Drosophila tumor suppressor gene dlg is required for normal synaptic bouton structure. Neuron 13:823-835. CrossRef Medline

Lee T, Luo L (1999) Mosaic analysis with a repressible cell marker for studies of gene function in neuronal morphogenesis. Neuron 22:451-461. CrossRef Medline

Li J, Ashley J, Budnik V, Bhat MA (2007) Crucial role of Drosophila neurexin in proper active zone apposition to postsynaptic densities, synaptic growth, and synaptic transmission. Neuron 55:741-755. CrossRef Medline

Liebl FL, Werner KM, Sheng Q, Karr JE, McCabe BD, Featherstone DE (2006) Genome-wide P-element screen for Drosophila synaptogenesis mutants. J Neurobiol 66:332-347. CrossRef Medline

Lin DM, Goodman CS (1994) Ectopic and increased expression of Fasciclin II alters motoneuron growth cone guidance. Neuron 13:507-523. CrossRef Medline

Luo L, Liao YJ, Jan LY, Jan YN (1994) Distinct morphogenetic functions of similar small GTPases: Drosophila Dracl is involved in axonal outgrowth and myoblast fusion. Genes Dev 8:1787-1802. CrossRef Medline

Marrus SB, Portman SL, Allen MJ, Moffat KG, DiAntonio A (2004) Differential localization of glutamate receptor subunits at the Drosophila neuromuscular junction. J Neurosci 24:1406-1415. CrossRef Medline

Mendoza-Topaz C, Urra F, Barría R, Albornoz V, Ugalde D, Thomas U, Gundelfinger ED, Delgado R, Kukuljan M, Sanxaridis PD, Tsunoda S, Ceriani MF, Budnik V, Sierralta J (2008) DLGS97/SAP97 is developmentally upregulated and is required for complex adult behaviors and synapse morphology and function. J Neurosci 28:304-314. CrossRef Medline

Meyer G, Varoqueaux F, Neeb A, Oschlies M, Brose N (2004) The complexity of PDZ domain-mediated interactions at glutamatergic synapses: a case study on neuroligin. Neuropharmacology 47:724-733. CrossRef Medline

Missler M, Fernandez-Chacon R, Südhof TC (1998) The making of neurexins. J Neurochem 71:1339-1347. Medline

Missler M, Zhang W, Rohlmann A, Kattenstroth G, Hammer RE, Gottmann K, Südhof TC (2003) Alpha-neurexins couple Ca2 + channels to synaptic vesicle exocytosis. Nature 423:939-948. CrossRef Medline

Mosca TJ, Hong W, Dani VS, Favaloro V, Luo L (2012) Trans-synaptic Teneurin signalling in neuromuscular synapse organization and target choice. Nature 484:237-241. CrossRef Medline

Nam CI, Chen L (2005) Postsynaptic assembly induced by neurexinneuroligin interaction and neurotransmitter. Proc Natl Acad Sci U S A 102:6137-6142. CrossRef Medline

Nourry C, Grant SG, Borg JP (2003) PDZ domain proteins: plug and play! Sci STKE 2003:RE7.

Parks AL, Cook KR, Belvin M, Dompe NA, Fawcett R, Huppert K, Tan LR, Winter CG, et al. (2004) Systematic generation of high-resolution deletion coverage of the Drosophila melanogaster genome. Nat Genet 36: 288-292. CrossRef Medline
Peng J, Kim MJ, Cheng D, Duong DM, Gygi SP, Sheng M (2004) Semiquantitative proteomic analysis of rat forebrain postsynaptic density fractions by mass spectrometry. J Biol Chem 279:21003-21011. CrossRef Medline

Prange O, Wong TP, Gerrow K, Wang YT, El-Husseini A (2004) A balance between excitatory and inhibitory synapses is controlled by PSD-95 and neuroligin. Proc Natl Acad Sci U S A 101:13915-13920. CrossRef Medline

Saus E, Brunet A, Armengol L, Alonso P, Crespo JM, Fernandez-Aranda F, Guitart M, Martin-Santos R, (2010) Comprehensive copy number variant $(\mathrm{CNV})$ analysis of neuronal pathways genes in psychiatric disorders identifies rare variants within patients. J Psychiatr Res 44:971-978.

Scheiffele P, Fan J, Choih J, Fetter R, Serafini T (2000) Neuroligin expressed in nonneuronal cells triggers presynaptic development in contacting axons. Cell 101:657-669. CrossRef Medline

Song JY, Ichtchenko K, Südhof TC, Brose N (1999) Neuroligin 1 is a postsynaptic cell-adhesion molecule of excitatory synapses. Proc Natl Acad Sci U S A 96:1100-1105. CrossRef Medline

Stewart BA, Atwood HL, Renger JJ, Wang J, Wu CF (1994) Improved stability of Drosophila larval neuromuscular preparations in haemolymphlike physiological solutions. J Comp Physiol A 175:179-191. CrossRef Medline

Südhof TC (2008) Neuroligins and neurexins link synaptic function to cognitive disease. Nature 455:903-911. CrossRef Medline

Sun M, Xing G, Yuan L, Gan G, Knight D, With SI, He C, Han J, Zeng X, Fang M, Boulianne GL, Xie W (2011) Neuroligin 2 is required for synapse development and function at the Drosophila neuromuscular junction. J Neurosci 31:687-699. CrossRef Medline

Szatmari P, Paterson AD, Zwaigenbaum L, Roberts W, Brian J, Liu XQ, Vincent JB, Skaug JL, et al. (2007) Mapping autism risk loci using genetic linkage and chromosomal rearrangements. Nat Genet 39:319-328. CrossRef Medline

Taniguchi H, Gollan L, Scholl FG, Mahadomrongkul V, Dobler E, Limthong N, Peck M, Aoki C, Scheiffele P (2007) Silencing of neuroligin function by postsynaptic neurexins. J Neurosci 27:2815-2824. CrossRef Medline

Thibault ST, Singer MA, Miyazaki WY, Milash B, Dompe NA, Singh CM, Buchholz R, Demsky M, et al. (2004) A complementary transposon tool kit for Drosophila melanogaster using P and piggyBac. Nat Genet 36:283287. CrossRef Medline

Varoqueaux F, Aramuni G, Rawson RL, Mohrmann R, Missler M, Gottmann K, Zhang W, Südhof TC, Brose N (2006) Neuroligins determine synapse maturation and function. Neuron 51:741-754. CrossRef Medline

Venken KJ, Carlson JW, Schulze KL, Pan H, He Y, Spokony R, Wan KH, Koriabine M, de Jong PJ, White KP, Bellen HJ, Hoskins RA (2009) Versatile P[acman] BAC libraries for transgenesis studies in Drosophila melanogaster. Nat Methods 6:431-434.

Wagh DA, Rasse TM, Asan E, Hofbauer A, Schwenkert I, Dürrbeck H, Buchner S, Dabauvalle MC, Schmidt M, Qin G, Wichmann C, Kittel R, Sigrist SJ, Buchner E (2006) Bruchpilot, a protein with homology to ELKS/ CAST, is required for structural integrity and function of synaptic active zones in Drosophila. Neuron 49:833-844. CrossRef Medline

Weyhersmüller A, Hallermann S, Wagner N, Eilers J (2011) Rapid active zone remodeling during synaptic plasticity. J Neurosci 31:6041-6052. CrossRef Medline

Wittenmayer N, Körber C, Liu H, Kremer T, Varoqueaux F, Chapman ER, Brose N, Kuner T, Dresbach T (2009) Postsynaptic Neuroligin1 regulates presynaptic maturation. Proc Natl Acad Sci U S A 106:13564-13569. CrossRef Medline

Woods DF, Bryant PJ (1991) The discs-large tumor suppressor gene of Drosophila encodes a guanylate kinase homolog localized at septate junctions. Cell 66:451-464. CrossRef Medline

Zeng X, Sun M, Liu L, Chen F, Wei L, Xie W (2007) Neurexin-1 is required for synapse formation and larvae associative learning in Drosophila. FEBS Lett 581:2509-2516. CrossRef Medline

Zhai RG, Bellen HJ (2004) The architecture of the active zone in the presynaptic nerve terminal. Physiology 19:262-270. CrossRef Medline 